\title{
RUNX1 prevents oestrogen-mediated AXIN1 suppression and $\beta$-catenin activation in ER-positive breast cancer
}

Nyam-Osor Chimge ${ }^{1,2}$, Gillian H. Little², Sanjeev K. Baniwal ${ }^{2}$, Helty Adisetiyo², Ying Xie ${ }^{3}$, Tian Zhang ${ }^{4}$, Andie O'Laughlin ${ }^{2}$, Zhi Y. Liu ${ }^{2}$, Peaches Ulrich², Anthony Martin'2, Paulette Mhawech-Fauceglia ${ }^{5}$, Matthew J. Ellis ${ }^{6}$, Debu Tripathy ${ }^{7}$, Susan Groshen ${ }^{8,9}$, Chengyu Liang ${ }^{4}$, Zhe Li $^{3}$, Dustin E. Schones ${ }^{10}$ \& Baruch Frenkel $2,9,11,12$

Recent high-throughput studies revealed recurrent RUNX1 mutations in breast cancer, specifically in oestrogen receptor-positive $\left(\mathrm{ER}^{+}\right)$tumours. However, mechanisms underlying the implied RUNX1-mediated tumour suppression remain elusive. Here, by depleting mammary epithelial cells of RUNX1 in vivo and in vitro, we demonstrate combinatorial regulation of AXIN1 by RUNX1 and oestrogen. RUNX1 and ER occupy adjacent elements in AXIN1's second intron, and RUNX1 antagonizes oestrogen-mediated AXIN1 suppression. Accordingly, RNA-seq and immunohistochemical analyses demonstrate an ER-dependent correlation between RUNX1 and AXIN1 in tumour biopsies. RUNX1 loss in ER ${ }^{+}$mammary epithelial cells increases $\beta$-catenin, deregulates mitosis and stimulates cell proliferation and expression of stem cell markers. However, it does not stimulate LEF/TCF, c-Myc or CCND1, and it does not accelerate G1/S cell cycle phase transition. Finally, RUNX1 loss-mediated deregulation of $\beta$-catenin and mitosis is ameliorated by AXIN1 stabilization in vitro, highlighting AXIN1 as a potential target for the management of $\mathrm{ER}^{+}$breast cancer.

\footnotetext{
${ }^{1}$ Department of Medicine, Keck School of Medicine of the University of Southern California, Los Angeles, California 90033, USA. ${ }^{2}$ Institute for Genetic Medicine, Keck School of Medicine of the University of Southern California, Los Angeles, California 90033, USA. ${ }^{3}$ Division of Genetics, Department of Medicine, Brigham and Women's Hospital, Harvard Medical School, Boston, Massachusetts 02115, USA. ${ }^{4}$ Department of Molecular Microbiology and Immunology, Keck School of Medicine of the University of Southern California, Los Angeles, California 90033, USA. ${ }^{5}$ Department of Pathology, Keck School of Medicine of the University of Southern California, Los Angeles, California 90033, USA. ${ }^{6}$ Smith Breast Center, Baylor College of Medicine, Houston, Texas 77030, USA. 7 Department of Breast Medical Oncology, The University of Texas MD Anderson Cancer Center, Houston, Texas 77030, USA. ${ }^{8}$ Department of Preventive Medicine, Keck School of Medicine of the University of Southern California, Los Angeles, California 90033, USA. ${ }^{9}$ USC/Norris Comprehensive Cancer Center, Keck School of Medicine of the University of Southern California, Los Angeles, California 90033, USA. ${ }^{10}$ Department of Diabetes Complications and Metabolism, Beckman Research Institute, City of Hope, Duarte, California 91010, USA. ${ }^{11}$ Department of Orthopedic Surgery, Keck School of Medicine of the University of Southern California, Los Angeles, California 90033, USA. ${ }^{12}$ Department of Biochemistry and Molecular Biology, Keck School of Medicine of the University of Southern California, Los Angeles, California 90033, USA. Correspondence and requests for materials should be addressed to N.C. (email: chimgee@usc.edu).
} 
B eside their developmental roles, particularly in haematopoiesis, skeletogenesis and neurogenesis, the three mammalian RUNX transcription factors have been assigned both oncogenic and tumour suppressor functions in a variety of neoplastic diseases ${ }^{1-3}$. In breast cancer, RUNX3 is frequently inactivated by promoter hypermethylation or protein mislocalization, its expression inversely correlates with disease progression $^{4,5}$, and its haploinsufficiency in mice promotes mammary ductal carcinoma ${ }^{6}$. Mechanistically, RUNX3 (as well as RUNX2) antagonize $\mathrm{ER} \alpha^{6-9}$. RUNX2, however, is better known for its pro-metastatic activity in breast and other carcinomas $^{3,10}$. Little attention has been paid thus far to the potential roles of RUNX1 in breast cancer. Recent studies, however, demonstrate that it is the predominant RUNX family member expressed in mammary epithelial cells ${ }^{2}$, and growing evidence suggests context-dependent dual roles for RUNX1 in breast cancer progression ${ }^{2,11-17}$. In particular, three independent studies of breast cancer patient cohorts have recently reported recurrent somatic mutations and/or deletions of RUNX1, as well as $C B F B$ that encodes an obligate co-activator of RUNX1 (refs 18-20). Here, we demonstrate that RUNX1 antagonizes oestrogen-mediated inhibition of AXIN1 expression, shedding light on its breast cancer suppression role.

Nearly two-thirds of all breast cancer cases belong to the ER ${ }^{+}$ luminal subtype ${ }^{21} . \mathrm{ER} \alpha$, which plays important physiological roles in mammary epithelial cell growth and differentiation during puberty and pregnancy, can acquire deleterious functions that promote breast carcinogenesis ${ }^{22-24}$. This is associated with changes to $E R \alpha$-mediated transcriptional stimulation or repression, attributable, in part, to increased ER $\alpha$ levels or alterations to modifying transcription factors such as FOXA, GATA, AP2 $\gamma$ and their associated co-regulators ${ }^{25-28}$. The present work calls attention to the ER $\alpha$-interacting transcription factor RUNX1 (ref. 29). It suggests that loss of RUNX1 in breast cancer facilitates $E R \alpha$-mediated suppression of $A X I N 1$, resulting in aberrant $\beta$-catenin signalling.

$\beta$-Catenin plays pivotal roles in cancer, primarily attributable to its role in canonical Wnt signalling. Upon Wnt pathway stimulation, a constitutively active $\beta$-catenin destruction complex is disassembled, allowing $\beta$-catenin to accumulate and ultimately activate LEF/TCF target genes such as CCND1, c-Myc, AXIN2 and $L E F 1$ itself $^{30,31}$. The $\beta$-catenin destruction complex contains, among others, the scaffold proteins AXIN1 and APC (adenomatous polyposis coli), as well as glycogen synthase kinase $3 \alpha / \beta \quad(\mathrm{GSK} 3 \alpha / \beta)$, which phosphorylate and mark $\beta$-catenin for proteasomal degradation ${ }^{30,32}$. In addition, $\beta$-catenin resides in the centrosome, where it regulates microtubule dynamics and bipolar mitotic spindle formation ${ }^{33-35}$. At the centrosome, $\beta$-catenin is phosphorylated by another kinase, NEK2, but is protected from degradation ${ }^{36}$. Despite its established oncogenic role in general, several issues regarding the role of $\beta$-catenin in $\mathrm{ER}^{+}$breast cancer remain to be elucidated. For instance, expression of $\beta$-catenin/TCF-regulated genes, both endogenous Wnt targets and reporter constructs, is poorly correlated with Wnt-driven mammary epithelial cell transformation that occur either spontaneously or experimentally ${ }^{37,38}$. In particular, increased expression of $c-M y c$ and CCND1, implicated in Wnt-driven loss of G1/S cell cycle control in colon cancer, is either absent or dispensable in many cases of $\mathrm{ER}^{+}$breast cancer ${ }^{37,38}$. Furthermore, it is unclear if and how oestrogen signalling may regulate $\beta$-catenin in breast cancer.

In this study, by depleting RUNX1 in vitro and in vivo, we expose a link between oestrogen and $\beta$-catenin in $\mathrm{ER}^{+}$breast cancer, that is, RUNX1-gated oestrogen-mediated AXIN1 transcriptional repression. Furthermore, we present evidence that deregulation of $\beta$-catenin in RUNX1-deficient ER ${ }^{+}$breast cancer cells is associated with compromised mitotic checkpoint control, accelerated cell proliferation and increased expression of stem cell markers. Our work marks AXIN1 as a potential therapeutic target to remedy deregulation of $\beta$-catenin in $\mathrm{ER}^{+}$breast cancer tumours that have lost RUNX1 function through somatic mutations or other mechanisms.

\section{Results}

RUNX1 loss deregulates $\boldsymbol{\beta}$-catenin. Expression of RUNX1 in the breast cancer cohort of The Cancer Genome Atlas (TCGA) ${ }^{20}$ varied considerably among individual tumours, with strong dependence on tumour subtype (Fig. 1a). RUNX1 mutations, mostly in the Runt DNA-binding domain ${ }^{2}$, were identified in 18 of the overall 524 tumours in this cohort, and 17 of them were within the group of $406 \mathrm{ER}^{+}$tumours ${ }^{20}$. In pursuit of molecular mechanisms contributing to its implied tumour suppressor activity in $\mathrm{ER}^{+}$breast cancer, we performed pathway analysis of genes differentially expressed in the $\mathrm{ER}^{+}$tumours with versus without RUNX1 mutations in TCGA, as well as in the ER ${ }^{+}$breast cancer cohort of Ellis et.al. ${ }^{18}$ Annotations associated with genes differentially expressed in each of these cohorts (Fig. 1b,c) or in both cohorts (Supplementary Fig. 1) were most significantly related to $\mathrm{Wnt} / \beta$-catenin signalling. The three gene lists and their inclusion criteria are provided in Supplementary Data 1-3, respectively.

Prompted by the data mining results, we next assessed the effect of RUNX1 silencing on $\beta$-catenin in the MCF7 and T47D cell culture models. Both of them represent the $\mathrm{ER}^{+}$luminal A breast cancer subtype, in which RUNX1 is most highly expressed (Fig. 1a), attributable in part to promoter hypomethylation (Supplementary Fig. 2). RUNX1 silencing with shRNAs that target either its RUNT domain $\left(\operatorname{shRx} 1_{\text {RUNT }}\right)$ or its $3^{\prime}$-untranslated region ( $3^{\prime}$-UTR; $\left.\operatorname{shRx} 1_{3^{\prime} \text {-UTR }}\right)$ upregulated active $\beta$-catenin (A- $\beta$-cat) levels in both cell lines (Fig. 1d), and increased cytoplasmic and nuclear $\beta$-catenin was confirmed by western blot analysis of the respective MCF7 cell fractions (Fig. 1e).

RUNX1 loss promotes cell growth and stem cell markers. Deregulation of $\beta$-catenin has been linked to cancer cell proliferation in general and cancer stem cells in particular ${ }^{39-41}$. Accordingly, RUNX1 knockdown with either $\operatorname{shRx} 1_{\mathrm{RUNT}}$ or shRx1 $1_{3^{\prime}-\text { UTR }}$ resulted in increased MCF7 breast cancer cell proliferation (Fig. 2a). Furthermore, conditional re-expression of RUNX1 in $\mathrm{MCF} / \mathrm{shRx} 1_{3^{\prime} \text {-UTR }}$ cells using a dox-inducible system normalized their growth rate (Fig. 2b). In addition, RUNX1 silencing was associated with upregulation of the stem cell markers ALDH1A3, CD44, AXIN2, NANOG and SOX2 (refs 42-46; Fig. 2c), and RUNX1 restoration normalized SOX2 mRNA levels (Fig. 2d). Similarly in T47D breast cancer cells, RUNX1 knockdown increased cell growth rate $^{13}$ and SOX2 expression (Fig. 2e). Moreover, mRNA profiles of Runx1-deficient mammary luminal epithelial cells from MMTV-Cre;Runx $1^{f / f}$ versus control mice (GSE 47377) indicated increased expression of Sox2 in response to Runx1 loss (Fig. 2f). Finally, as shown in Fig. $2 \mathrm{~g}$, SOX2 mRNA was markedly elevated in biopsies from RUNX1-mutant versus RUNX1-WT human primary breast tumours in the clinical cohort of Ellis et al. ${ }^{18}$ Thus, deregulation of $\beta$-catenin in RUNX1-deficient $\mathrm{ER}^{+}$breast cancer might contribute to disease progression by promoting cell growth in general and expansion of a stem cell-like population in particular.

RUNX1 regulates $A X I N 1$. In pursuit of RUNX1 target genes in $\mathrm{ER}^{+}$breast cancer, which may mediate the regulation of $\beta$-catenin levels, we determined both the RUNX1 transcriptome 

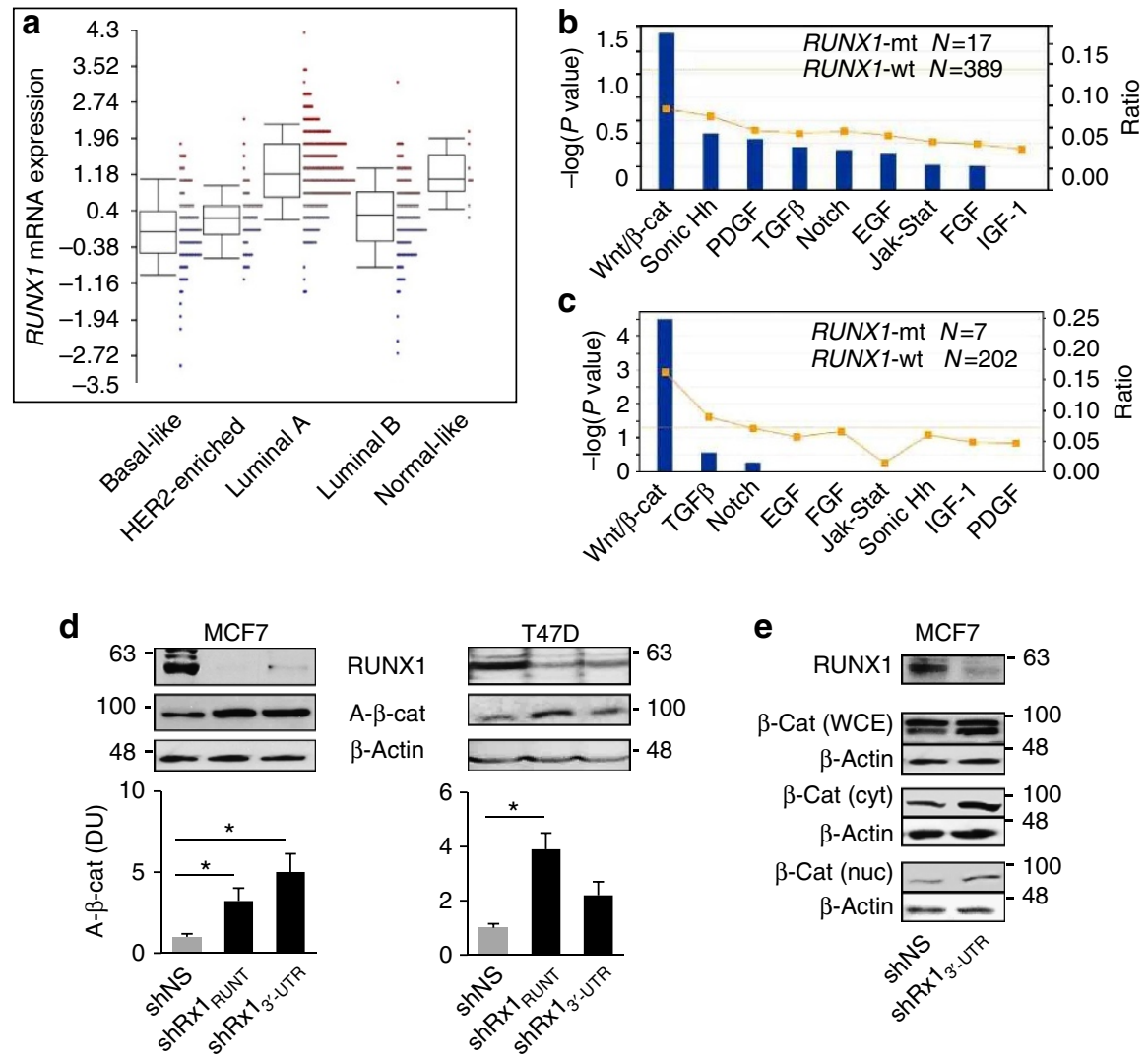

Figure 1 | Upregulation of $\boldsymbol{\beta}$-catenin in RUNX1-deficient breast cancer. (a) RUNX1 mRNA expression in the five major breast cancer subtypes in the breast cancer cohort of TCGA ${ }^{20}$. Expression levels are significantly different between the subtypes $(P=6.8 \mathrm{e}-38$ by analysis of variance). Boxes represent the $25 \%$ to $75 \%$ quartiles, lines within boxes represent the median levels and whiskers represent the non-outlier range. (b) Genes differentially expressed in $\mathrm{ER}^{+}$tumours with mutant versus wild-type RUNX1 in the breast cancer patient cohort of TCGA ${ }^{20}$ (Supplementary Data 1) were interrogated using Ingenuity Pathways Analysis (IPA) for annotations related to major developmental signalling pathways. Line graph represents fold enrichment, and statistical significance (bars) was calculated by Fisher's exact test as implemented in the IPA software. (c) IPA analysis was performed as in $\mathbf{b}$ for the differentially expressed genes (Supplementary Data 2) in RUNX1-mutant tumours in the breast cancer patient cohort of Ellis et al. ${ }^{18}$ (d) Top: representative western blot analyses of the indicated proteins in MCF7 and T47D cells expressing either a nonspecific shRNA (shNS) or shRNAs targeting the Runt

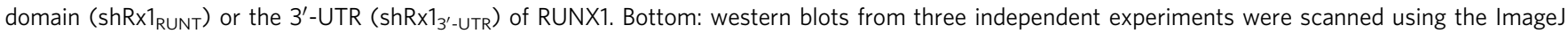
software, and bar graphs represent mean densitometric values ( \pm s.e.m.) for normalized A- $\beta$-cat corrected for $\beta$-actin. ${ }^{\star} P<0.05$ by $t$-test. (e) Western blot analysis of total $\beta$-catenin in whole-cell extracts (WCE), as well as cytoplasmic (cyt) and nuclear (nuc) fractions of MCF7 cells expressing the shNS or the ShRx1 $1_{3^{\prime}-U T R}$ RNAs.

and its cistrome in MCF7 cells by mRNA profiling and ChIP-seq analysis, respectively. We first compared global mRNA expression in cells expressing $\operatorname{shRx} 1_{3^{\prime} \text {-UTR }}$ versus cells expressing a nonspecific hairpin RNA (shNS) as in Figs 1e and 2. Because recurrent RUNX1 mutations are specific to $\mathrm{ER}^{+}$tumours ${ }^{20}$, we determined the differentially expressed genes both in the presence and absence of estradiol (E2), and turned our attention to 599 genes that responded to RUNX1 knockdown in the presence of E2 (Fig. 3a, Supplementary Data 4). We then profiled RUNX1 locations with ChIP-seq to identify putative direct RUNX1 targets, those that not only respond to RUNX1 (in the presence of E2) but also physically associate with RUNX1. As shown in Fig. 3b, there was enrichment of RUNX1-occupied regions (R1ORs) near the transcription start sites (TSSs) of the RUNX1-responsive genes, likely related to short-range direct transcriptional regulation. We next interrogated an MCF7 ER $\alpha$ ChIP-seq data set $^{47}$ to identify putative RUNX1-responsive enhancers that also recruited $\mathrm{ER} \alpha$, potentially accounting for dependence of the RUNX1 response on oestrogen. Of the 176 R1ORs present between positions $-500 \mathrm{~kb}$ and $+500 \mathrm{~kb}$ relative to the TSSs of the 599 RUNX1-reponsive genes, 36 genes (named in Fig. $3 \mathrm{~b}$ ) were also occupied by $\mathrm{ER} \alpha$. Among the regions co-occupied by RUNX1 and ER $\alpha$ was the second intron of AXIN1
(Fig. 3b,c), encoding a pivotal regulatory component of the $\beta$-catenin destruction complex. Co-occupancy of this region by RUNX1 and ER $\alpha$ was validated by ChIP-quantitative PCR (ChIP-qPCR; Fig. 3d). RT-qPCR (qPCR with reverse transcription) analysis confirmed the downregulation of AXIN1 expression upon RUNX1 silencing (Fig. 3e). Furthermore, conditional induction (by dox treatment) of wild-type RUNX1, but not RUNX1 mutants with the amino acid substitutions D198G or R166Q found in human breast cancers ${ }^{18,20}$, restored AXIN1 mRNA expression (Fig. 3e).

RUNX1 prevents oestrogen-mediated inhibition of $A X I N 1$. We next addressed the dependence of the RUNX1-AXIN1 axis on oestrogen signalling using cell and animal experimental models, as well as clinical data mining. First, we assessed by RT-qPCR the effect of dox-mediated RUNX1 silencing on AXIN1 expression in MCF7/shRx $1^{\text {dox }}$ cultures maintained in charcoal-stripped serum (CSS) with or without added E2. As shown in Fig. 4a, RUNX1 silencing in the absence of oestrogens did not itself affect AXIN1 expression. However, RUNX1 silencing in the presence of E2 resulted in the suppression of AXIN1. Second, we tested the dependence of the RUNX1-AXIN1 axis on oestrogens in 
a

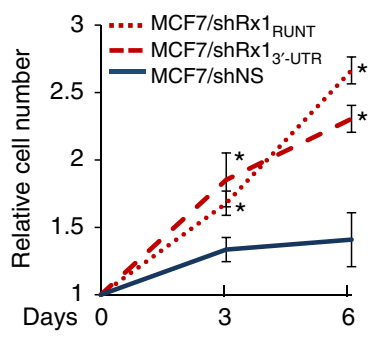

b

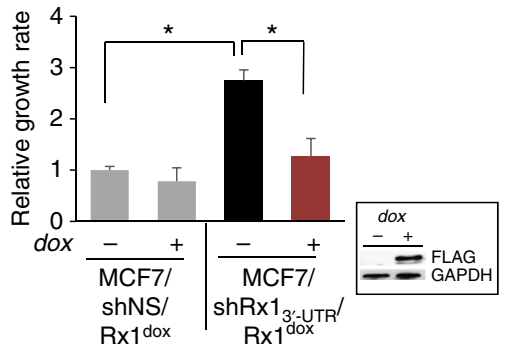

C MCF7

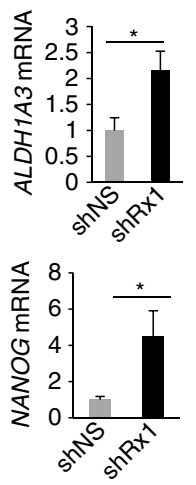

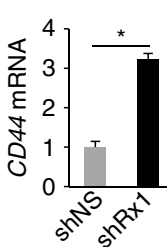

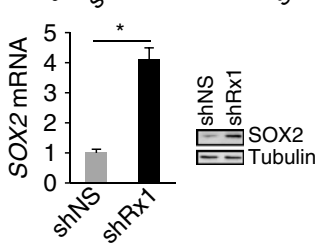

d

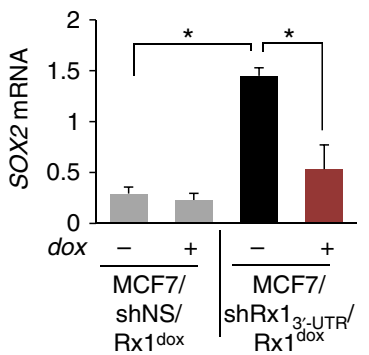

e
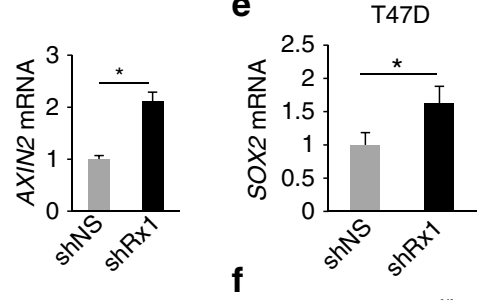

MMTV-Cre;Runx ${ }^{t f f} ;$ R26Y

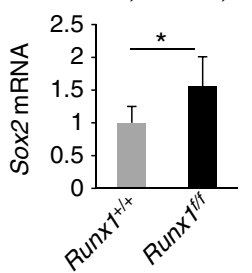

g Tumor biopsies

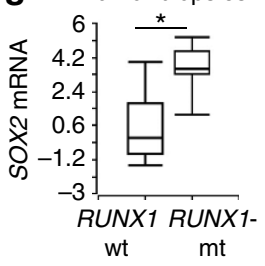

Figure 2 | Increased proliferation and expression of stem cell markers in RUNX1-depleted mammary epithelial cells in vitro and in vivo. (a) MCF7 cells expressing nonspecific (NS) or the indicated RUNX1-targeting shRNAs were plated and their growth rate was assessed by MTT assays on days 3 and 6. (b) RUNX1 was silenced with shRx1 $3_{3^{\prime}-U T R}$ as in Fig. 1d,e, and was re-expressed from a dox-inducible vector as demonstrated by the western blot in the inset. Cell growth was assessed as in $\mathbf{a}$ and bars represent the increase in MTT values between day 3 and day 6. (c) MCF7 cells expressing a nonspecific shRNA (shNS) or shRx1 $1_{3^{\prime}-\text { UTR }}$ were subjected to RT-qPCR analysis of the indicated stem cell markers. Expression of Sox2 was also assessed by western blot analysis. (d) RT-qPCR analysis of SOX2 in MCF7 cells in which RUNX1 was silenced and then restored as in $\mathbf{b}$. (e-g) Comparisons of SOX2 mRNA expression between (e) RUNX1-depleted versus control T47D cells; (f) mammary luminal epithelial cells from MMTV-Cre;Runx $f / f$;R26Y versus control MMTV-Cre;RunX $1^{+/+} ; R 26 Y$ mice based on our microarray data in GSE47377 (ref. 13); (g) RUNX1-mutant $(n=7)$ versus $R U N X 1-$ WT ( $\left.n=202\right)$ breast cancer tumours based on our microarray database ${ }^{18}$, where boxes represent the 25 th-75th percentile range, horizontal lines within boxes represent the median values and whiskers extend to the minimum and maximum values. Where applicable, data represent mean \pm s.e.m. of triplicate experiments. ${ }^{\star} P<0.05$ by $t$-test (a-f) or by Mann-Whitney test $(\mathbf{g})$.

$\mathrm{MCF} 7 / \mathrm{shRx} 1^{\text {dox }}$ cells cultured in complete (oestrogen-containing) serum (as in Figs 1 and 2) with or without the ER antagonist/ downregulator ICI 182780. As shown in Fig. 4b, dox-mediated RUNX1 silencing resulted in decreased AXIN1 expression in the absence but not in the presence of ICI 182780. These results suggest that AXIN1 is negatively regulated by oestrogen signalling, and that this negative regulation is denied by RUNX1 (Fig. 4a,b). Notably, the combinatorial regulation of AXIN1 by RUNX1 and E2 is unusual, because the global transcriptional response to RUNX1 knockdown is generally independent on E2 (Fig. 4c). In addition, unlike the global transcriptional response to RUNX2 overexpression ${ }^{7,9,48}$, the global transcriptional response to RUNX1 overexpression is generally independent of E2 (Supplementary Fig. 3).

We further examined the dependence of the RUNX1-AXIN1 axis on oestrogen signalling in vitro by comparing the effect of dox-mediated RUNX1 silencing on AXIN1 expression in $\mathrm{ER}^{+}$ versus $\mathrm{ER}^{-}$mammary epithelial cell lines. As demonstrated by western blot analysis, dox-mediated silencing of RUNX1 with either shRx1 $1_{3^{\prime} \text {-UTR }}$ (Fig. 4d) or shRx1 $1_{\text {RUNT }}$ (Supplementary Fig. 4) resulted in the downregulation of AXIN1 expression in the ER ${ }^{+}$ MCF7 and T47D cells, but not in the ER ${ }^{-}$MDA-MB-231 or MCF10A cells.

We next set to test the effect of Runxl on Axin1 mRNA expression in $\mathrm{ER}^{+}$versus $\mathrm{ER}^{-}$murine mammary epithelial cells in vivo. Our strategy was to isolate mature luminal cells (ML; predominantly ER-positive) and luminal progenitor cells
(LP; predominantly ER-negative) from MMTV-Cre;Runx $1^{f / f}$ and control mice, and compare the two cell populations in terms of the effect of Runx1 ablation on Axin1 expression. However, because, as reported previously ${ }^{13}$, the ML cell population was virtually lost in MMTV-Cre;Runxilf mice, we isolated Runx1-depleted and control ML cells from mice with additional ablation of $R b 1$, in which the ML cell population was restored despite the absence of Runx1. As shown in Fig. 4e, Runx1 ablation resulted in decreased Axin1 expression in the predominantly $\mathrm{ER}^{+} \mathrm{ML}$ cells but not in the predominantly ER $\mathrm{LP}^{-}$cells, again suggesting E2-dependent regulation of AXIN1 by RUNX1. Thus, multiple experimental approaches in vitro and in vivo indicate combinatorial regulation of AXIN1 by RUNX1 and oestrogens in both normal and transformed mammary epithelial cells. These results implicate AXIN1 suppression in E2-driven breast carcinogenesis, containment of which accounts for the tumour suppressor activity of RUNX1 in $\mathrm{ER}^{+}$breast cancer. Dependence of the RUNX1-AXIN1 axis on oestrogens may explain the observation of recurrent RUNX1 somatic mutations in $\mathrm{ER}^{+}$but not $\mathrm{ER}^{-}$breast cancer tumours ${ }^{20}$.

Association between RUNX1 and AXIN1 in $\mathrm{ER}^{+}$breast cancer. To address the combinatorial regulation of AXIN1 by RUNX1 and oestrogens in clinical settings, we first calculated an inhibitory index' for RUNX1 in each tumour in the breast cancer cohort of TCGA ${ }^{20}$ based on the expression levels of genes that 
a

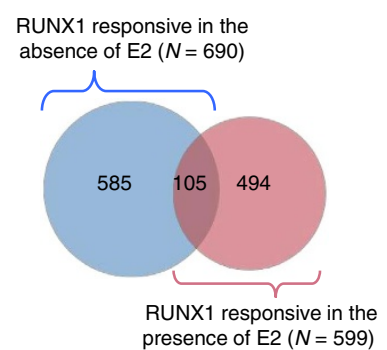

C

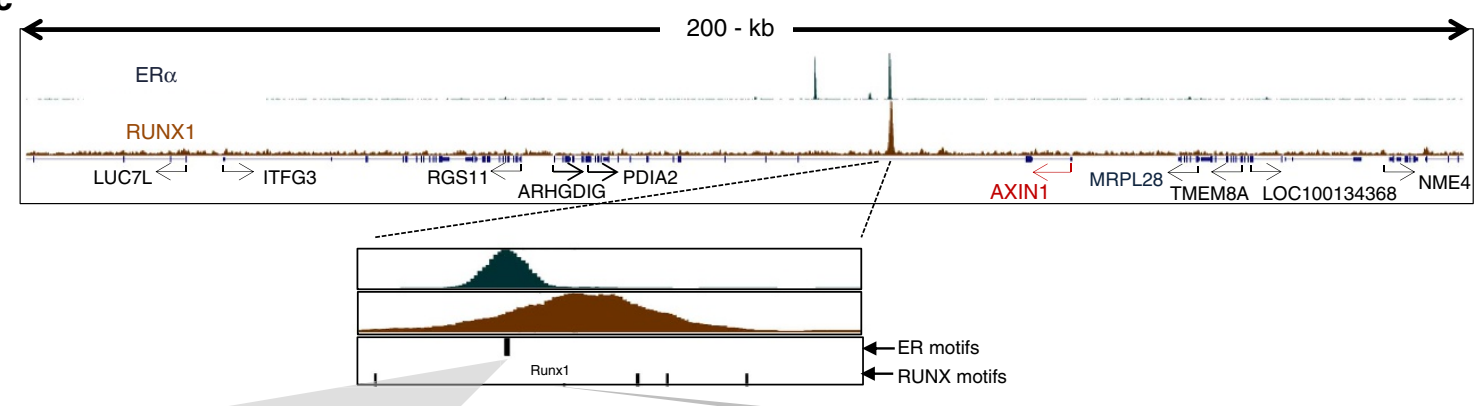

b 7

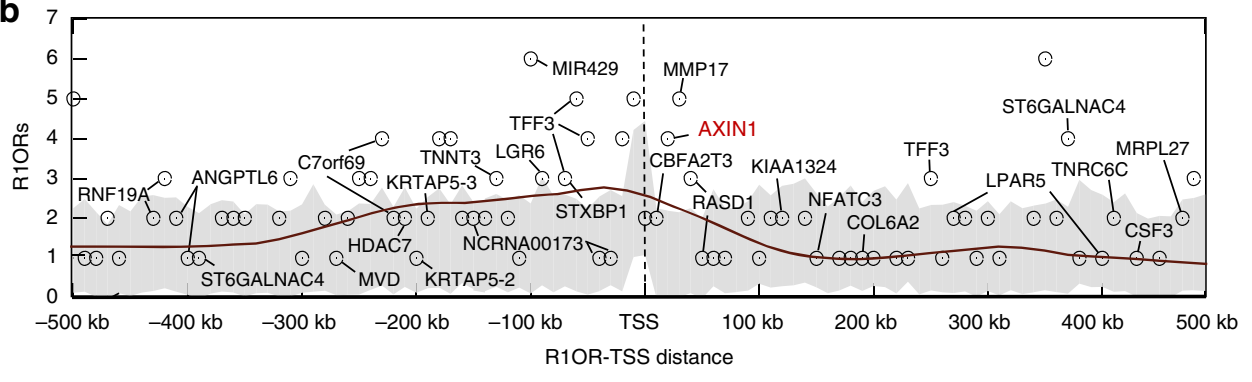

CGCTGTGGTTCC
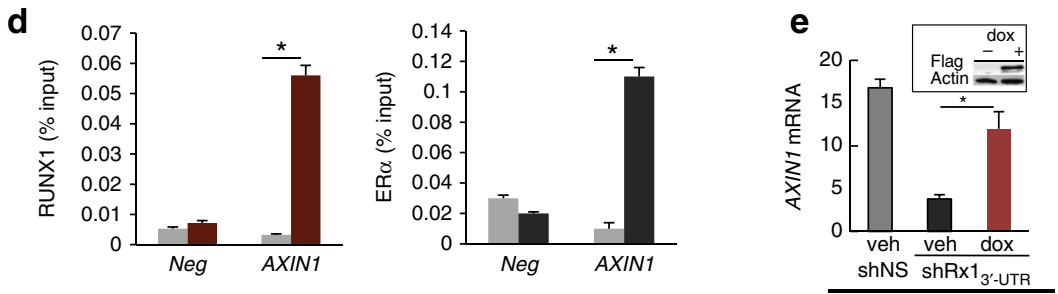

MCF7/Rx1 dox
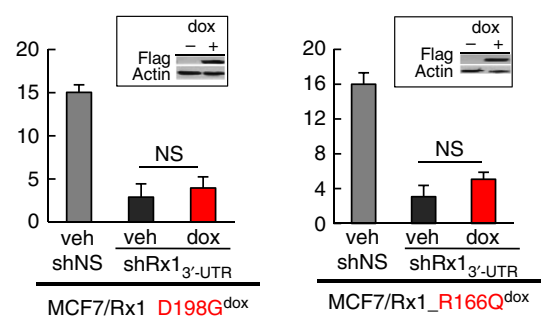

Figure 3 | Recruitment of RUNX1 and ER $\boldsymbol{\alpha}$ to adjacent elements in AXIN1. (a) The genome-wide response to RUNX1 was determined as described in the 'Methods' section by comparing the mRNA profiles of MCF7/shRx1 $1_{3^{\prime}-U T R}$ and MCF7/shNS cells maintained in CSS \pm E2. The Venn diagram describes the RUNX1 responsiveness (number of genes with fold change $>1.5$ and $P<0.05$ ) in the presence and absence of E2. (b) RUNX1-occupied regions (R1ORs) were determined by ChIP-seq analysis of MCF7 cells and mapped with respect to the TSSs of the 599 genes that responded to RUNX1 in the presence of E2. For 111 out of these 599 genes, R1ORs were found between positions $-500 \mathrm{~kb}$ and $+500 \mathrm{~kb}$ of the respective TSSs, and these R1ORs (a total of 176) were enumerated in 10-kb bins. Grey area represents the results of 1,000 iterations with control random gene sets. The brown line is a density curve of R1OR occurrence generated by LOESS fitting to R1OR counts $(\alpha=0.25)$. Gene names are depicted in cases where R1ORs overlapped with ER-occupied regions deduced from GSE14644 (ref. 47). (c) Screen shots of RUNX1 and ER $\alpha$ ChIP-seq data for the AXIN1 locus, with zoom-in on ER $\alpha$ and RUNX1 peaks at a region co-occupied by the two transcription factors. (d) ChIP-qPCR confirmation of RUNX1 and ER $\alpha$ occupancy at the second intron of AXIN1. Data obtained with antibodies against RUNX1 (left) and ER $\alpha$ (right) are represented by brown and black bars, respectively, and data obtained with control lgG is represented by grey bars. ${ }^{\star} P<0.05$ by $t$-test. (e) Endogenous $R U N X 1$ in MCF7 cells was silenced by shRx $1_{3^{\prime} \text {-UTR }}$ and the indicated wild-type or mutant FLAG-tagged RUNX1 proteins were induced by dox as in Fig. 2b,d. AXIN1 expression was assessed by RT-qPCR. Data was corrected for 18S RNA and represent mean \pm s.e.m. from triplicate experiments. ${ }^{\star} P<0.05$ by $t$-test. Insets represent western blot analyses of the exogenous $F$ LAG-tagged proteins.

RUNX1 inhibited in MCF7 cells (see 'Methods' section). As shown in Fig. 5a, AXIN1 mRNA significantly correlated with the RUNX1 inhibitory index in $\mathrm{ER}^{+}$but not in the $\mathrm{ER}^{-}$breast cancer types. We further analysed the association between AXIN1 and RUNX1 at the protein level using a commercial tumour microarray, TMA-1007, which included among others duplicate cores from $31 \mathrm{ER}^{+}$invasive ductal carcinomas in which ER was expressed at either low $\left(\mathrm{ER}^{\mathrm{low}}\right)$ or high levels (ER high). We immunostained the TMA to define each tumour as positive or negative for RUNX1 and AXIN1. Despite the reported scarcity of RUNX1 mutations ( $\leq 5 \%$ in three large independent studies $^{18-20}$ ), RUNX1 was undetectable in 12 of the $31 \mathrm{ER}^{+}$ ductal invasive carcinomas, indicating that its function may be lost, at least transiently, in far more than the $\leq 5 \%$ of tumours with RUNX1 mutations (Supplementary Table 1). AXIN1 was undetectable in 11 of the 31 tumours, but there was no correlation between the RUNX1 and the AXIN1 status across all the tumours. However, examination of the correlation in relation to the ER levels demonstrated strong positive association between RUNX1 and AXIN1 (odds ratio of $21.7 ; P=0.033$ ) in the ER ${ }^{\text {high }}$ tumours with no significant correlation in the ER ${ }^{\text {low }}$ tumours (Fig. 5b and Supplementary Table 1). The positive correlation in the $\mathrm{ER}^{\text {high }}$ group is illustrated by representative immunohistochemical images in Fig. 5c. Given the evidence from cell culture and animal models for combinatorial regulation of AXIN1 by RUNX1 and oestrogens (Fig. 4), the analyses of the RNA-seq and the TMA data (Fig. 5) suggest ER-dependent regulation of AXIN1 by RUNX1 in patient tumours as well.

Abbreviated mitosis in RUNX1-depleted breast cancer cells. Activation of the Wnt/ $\beta$-Catenin pathway is traditionally thought to promote cell proliferation through LEF/TCF-mediated stimulation of target genes such as CCND1, c-MYC and LEF1 
a
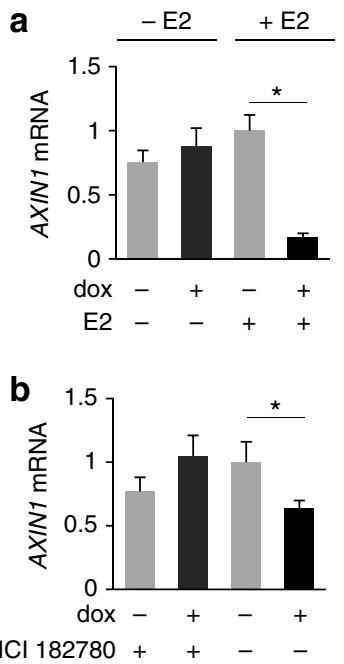

C

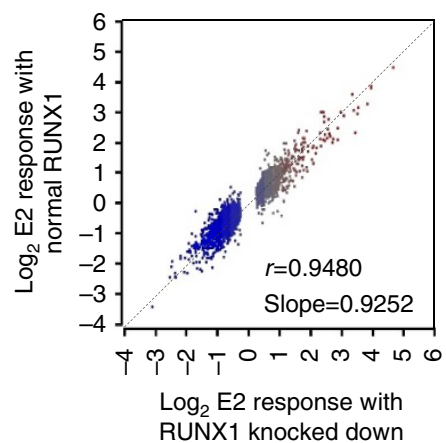

d

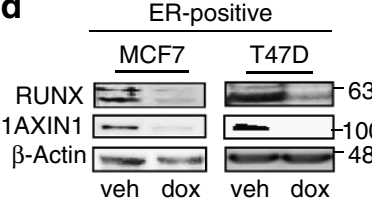

e

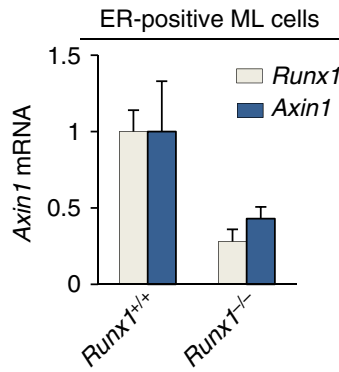

ER-negative

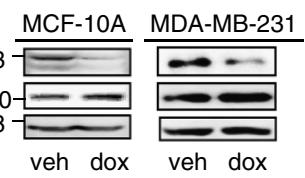

ER-negative LP cells

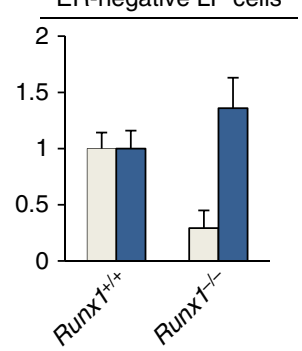

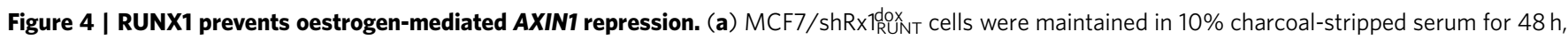
treated as indicated for the following $48 \mathrm{~h}$, and AXIN1 mRNA levels were measured by RT-qPCR and corrected for 18S RNA (mean \pm s.e.m. of three independent experiments). (b) MCF7/shRx dox RiNT cells in 10\% complete serum were treated as indicated for $48 \mathrm{~h}$, and AXIN1 mRNA levels were measured by RT-qPCR and corrected for $18 \mathrm{~S}$ RNA (mean \pm s.e.m. of three independent experiments). ${ }^{\star} P<0.05$ by $t$-test. (c) Scatter plot of the global E2 responsiveness in the presence ( $y$ axis) versus absence ( $x$ axis) of RUNX1 in MCF7 cells. (d) The indicated ER ${ }^{+}$(left) and ER ${ }^{-}$(right) mammary epithelial cell lines were engineered with the dox-inducible shRx1 $1_{3^{\prime} \text {-UTR }}$ lentiviral vector and treated with dox for 4 days before western blot analysis of the indicated proteins. (e) RT-qPCR results for Axin1 and Runx1 from predominantly ER ${ }^{+}$mature luminal (ML) mammary epithelial cells (left) and predominantly ER ${ }^{-}$ luminal progenitor (LP) cells (right) isolated from RUNX1-knockout and control mammary glands as described in the 'Methods' section.

itself, resulting in accelerated G1/S cell cycle transition ${ }^{39,49,50}$. However, cell cycle profiling showed that RUNX1 silencing, either constitutively or conditionally (by dox treatment), resulted in increased, not decreased percentage of cells in the G1 phase of the cell cycle (Fig. 6a,b). Accordingly, RUNX1 silencing increased the mRNA levels of neither $c-M y c$, nor CCND1 nor LEF1 (Fig. $6 \mathrm{c}$ and Supplementary Fig. 5), nor did it upregulate expression of the LEF/TCF TOPFLASH reporter (Fig. 6d). Furthermore, expression of neither $c-M y c, C C N D 1$, nor LEF1 differed in TCGA breast cancer tumours with wild-type versus mutant RUNX1 (Fig. 6e). Rather than accelerated G1/S transition, the cell cycle profiles (Fig. 6a,b) suggested abbreviated mitosis, indicated by a reproducible decrease in $\mathrm{G} 2 / \mathrm{M}$ cells, and this effect was observed with both complete (oestrogen-containing) medium (Fig. 6a) and after the supplementation of CSS with E2 (Fig. 6b).

To further investigate the role of RUNX1 in regulating breast cancer cell cycle progression, we challenged control and RUNX1depleted MCF7 cells by treatment with the anti-mitotic taxane drug docetaxel, which normally induces a G2/M block and a mitotic catastrophe ${ }^{51,52}$. We first confirmed (Fig. 6f) the accumulation of cells in G2/M, as well as the presence of cell debris, in naive cultures treated with $2 \mathrm{nM}$ docetaxel. A similar response was observed in MCF7/RUNX1 $1_{\mathrm{RUNT}}^{\text {dox }}$ cells cultured in the absence of dox as control (Fig. 6g, left). However, dox-treated (RUNX1-depleted) MCF7/RUNX1 RUN $_{\text {dox }}$ cells (Fig. 6g, right), and not dox-treated naive MCF7 cells used as control (Fig. 6f, right) were resistant to docetaxel. Consistent with abbreviated mitosis, cyclin B1 levels decreased in the RUNX1-depleted, docetaxeltreated compared with control cells (Fig. 6g, inset). Similar results were obtained with the microtubule-targeting agent nocodazole (Supplementary Fig. 6).

Mitotic abbreviation in RUNX1-depleted $\mathrm{ER}^{+}$breast cancer cells could result from deregulation of $\beta$-catenin at the centrosome, where it is locally phosphorylated and controls microtubule organization before and during mitosis ${ }^{33,34,36}$. Given its cell cycle-dependent expression ${ }^{53}$ (Supplementary Fig. 7), we measured phospho- $\beta$-catenin (P- $\beta$-cat) levels by western blot analysis of cells blocked at G1/S, G2/M or after release. As shown in Fig. 6h, RUNX1 depletion resulted in increased P- $\beta$-cat levels specifically around $8 \mathrm{~h}$ after release from a G1/S block. The cell cycle-dependent increase in P- $\beta$-cat, which is undetectable in unsynchronized cells (Supplementary Fig. 8) may account for deregulated mitosis in RUNX1-depleted $\mathrm{ER}^{+}$breast cancer cells through its role in the centrosome ${ }^{33-36}$ (Supplementary Fig. 9).

Partial restoration of cell cycle control by AXIN1 stabilization. Because RUNX1 loss resulted in decreased AXIN1, the least abundant component of the $\beta$-catenin destruction complex and a rate-limiting factor for $\beta$-catenin phosphorylation and degradation $^{32,54}$, we explored the effects the AXIN1 stabilizer IWR1 (ref. 55) on the deregulated cell cycle in RUNX1-depleted MCF7 cells. Remarkably, the IWR1-mediated restoration of AXIN1 and the subsequent normalization of $\beta$-catenin levels in MCF7/shRx1 cells (Fig. 7a) resulted in a decrease in cell growth rate (Fig. 7b) to levels measured in control MCF7/shNS cultures. Furthermore, IWR1 prevented the cell cycle-dependent increase in P- $\beta$-cat level (Fig. 7c) and restored docetaxel-mediated G2/M block (Fig. 7d) in dox-treated (RUNX1-depleted) MCF7/shRx1 ${ }^{\text {dox }}$ cells. Taken together, our results assign a role for RUNX1 in antagonizing oestrogen-mediated AXIN1 suppression and highlight AXIN1 as a potential target for the treatment of RUNX1-deficient $\mathrm{ER}^{+}$ breast cancer (Fig. 7e).

\section{Discussion}

This study demonstrates combinatorial regulation of AXIN1 by RUNX1 and oestrogen signalling in $\mathrm{ER}^{+}$breast cancer cells. AXIN1 mRNA and protein levels were decreased upon RUNX1 silencing, and this was observed only in the presence of oestrogen. It did not occur in (1) ER ${ }^{-}$breast epithelial cell lines (Fig. 4d); (2) ER ${ }^{+}$breast cancer cells treated with the ICI 182780 (Fig. 4b); and (3) $\mathrm{ER}^{+}$breast cancer cells cultured in CSS without added E2 (Fig. 4a). That RUNX1 regulates AXIN1 in an 

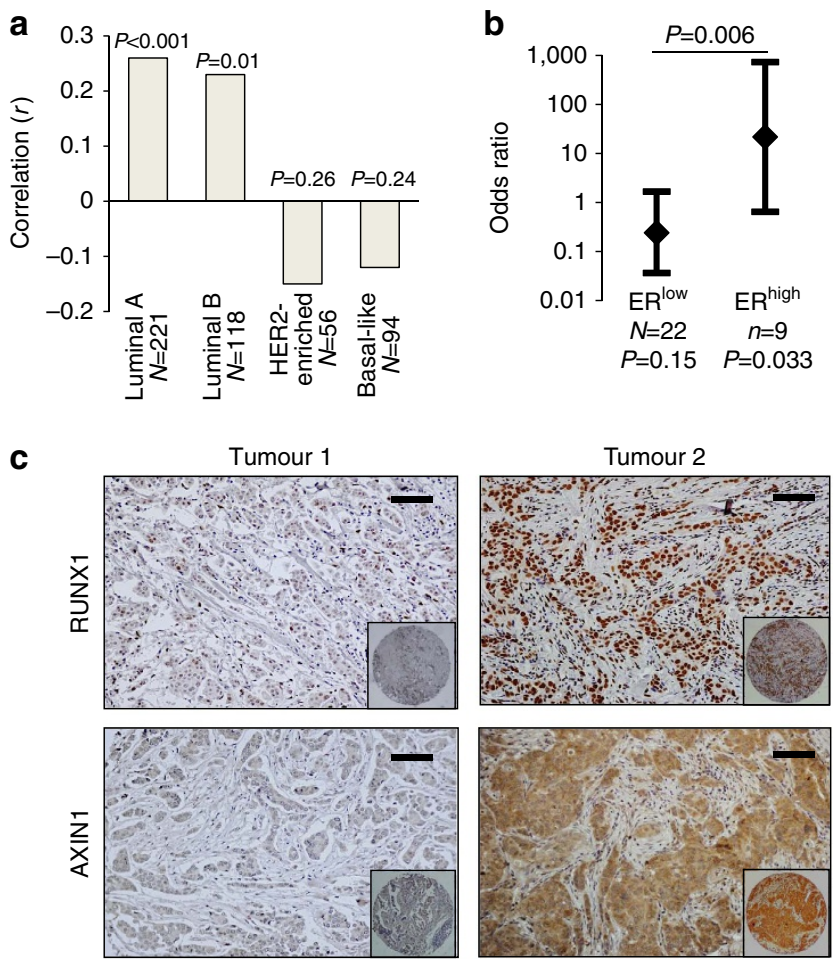

Figure 5 | Association between RUNX1 and AXIN1 in ER ${ }^{+}$breast cancer tumours. (a) Correlation between the RUNX1 inhibitory index and AXIN1 mRNA in the breast cancer patient cohort of TCGA (ref. 20). The RUNX1 inhibitory index was calculated for each tumour as described in the 'Methods' section and the correlation with AXIN1 mRNA (UCSC isoform uc002cgp.1) was calculated. Bars represent the Pearson linear correlations ( $r$ ) and $P$ values were determined based on $r$ and the sample size $N$. (b) Breast cancer tumour microarray TMA-1007 from Protein Biotechnologies, Inc. was immunostained for RUNX1 and AXIN1. The ER ${ }^{+}$ invasive ductal carcinomas were designated as positive or negative for RUNX1 and AXIN1. Data represent the odds ratio and the $95 \%$ confidence intervals for the association between AXIN1 status and RUNX1 status in tumours expressing ER $\alpha$ at either low (ER ${ }^{\text {low }}$ ) or high levels (ER high). Association between the RUNX1 status and AXIN1 status was tested using the Pearson chi-square test for the $2 \times 2$ table, for ER ${ }^{\text {low }}$ and ER ${ }^{\text {high }}$ tumours separately. Odds ratios for the ER ${ }^{\text {low }}$ and $E R^{\text {high }}$ tumours were compared using the Breslow-Day test for homogeneity of odds ratios. (c) RUNX1 and AXIN1 immunohistochemical staining of two ER high tumours from the TMA illustrating the association between RUNX1 and AXIN1 expression (Magnification $\times 20$; scale bar, $50 \mu \mathrm{m}$; insets show the $1.1 \mathrm{~mm}$ cores in their entirety).

oestrogen-dependent manner in vivo is suggested by (1) the decreased Axin 1 expression in the predominantly $\mathrm{ER}^{+} \mathrm{ML}$ cells, but not in the predominantly ER ${ }^{-}$LP cells in Runx1-deficient versus control murine mammary epithelium (Fig. 4e), (2) the positive correlation between AXIN1 mRNA and the RUNX1 inhibitory index in $\mathrm{ER}^{+}$but not $\mathrm{ER}^{-}$breast cancer subtypes in TCGA (Fig. 5a) and (3) the positive correlation between RUNX1 and AXIN1 protein levels in $\mathrm{ER}^{\text {high }}$ but not $\mathrm{ER}^{\text {low }}$ human $\mathrm{ER}^{+}$ breast cancer tumours (Fig. 5b). Importantly, RUNX1 did not stimulate AXIN1 expression in the absence of oestrogens; rather, it prevented oestrogen-mediated AXIN1 repression (Fig. 4). Such combinatorial regulation by RUNX1 and oestrogens does not represent genome-wide antagonism of oestrogen-regulated gene expression (Fig. 4c); instead, it likely reflects local interaction between ER $\alpha$ and RUNX1 at a co-occupied regulatory region in the second intron of AXIN1 (Fig. 3c). This co-occupancy does not seem to occur through a mechanism of tethering as described for forcibly expressed ER $\alpha$ in ER ${ }^{-}$MDA-MB-231 cells $^{29}$. Evidence arguing against recruitment of endogenous ER $\alpha$ by DNA-bound RUNX1 in MCF7 cells has been presented previously ${ }^{29}$ and is certainly unlikely at the AXIN1 locus, where the ER $\alpha$ and RUNX1 ChIP-seq peaks are phased, each perfectly aligned with its respective DNA sequence motif (Fig. 3c). Although genome-wide transcriptional regulation by RUNX1 is not generally dependent on oestrogens (Fig. 4c, Supplementary Fig. 3), such dependence at a few critical regulatory loci, such as $A X I N 1$, may explain the specificity of RUNX1 recurrent mutations to $\mathrm{ER}^{+}$breast cancer tumours ${ }^{18,20}$. While additional loci (for example, NCRNA00173, LGR6, TFF3 and CBFA2T3, MMP17; Fig. 3b) may also contribute to the oestrogen-dependent tumour suppressor activity of RUNX1 in breast cancer, the role of AXIN1 downstream of RUNX1 in this context is strongly supported by deregulation of $\beta$-catenin in RUNX1-deficient ER ${ }^{+}$breast cancer cells and the corrective effects of IWR1. Our data therefore demonstrates crosstalk between oestrogen and $\beta$-catenin signalling in $\mathrm{ER}^{+}$ breast cancer through AXIN1. It further suggests that RUNX1 suppresses $\mathrm{ER}^{+}$breast cancer progression by denying oestrogens their negative regulation of AXIN1.

Our work begins to elucidate tumour suppression mechanisms operative downstream of the RUNX1-AXIN1 axis in ER ${ }^{+}$breast cancer cells. As expected, loss of AXIN1 expression after RUNX1 silencing in the MCF7 and T47D cell lines was associated with increased $\beta$-catenin levels (Fig 1d,e). However, unlike colon cancer, increased $\beta$-catenin in breast cancer does not seem to deregulate the $\beta$-catenin/TCF-driven transcription in the canonical Wnt pathway ${ }^{37,38}$. Accordingly, the upregulation of $\beta$-catenin in RUNX1-depleted $\mathrm{ER}^{+}$breast cancer cell lines was not associated with increased expression of the LEF/TCFresponsive G1/S regulatory genes $C C N D 1$ and $c-M y c$ (Fig. 6c; Supplementary Fig. 5). Likewise, the Wnt reporter TOPFLASH was not stimulated (Fig. 6d) and G1/S cell cycle transition was not accelerated (Fig. 6a,b). Instead, E2-mediated AXIN1 suppression upon RUNX1 loss may contribute to $\mathrm{ER}^{+}$breast cancer progression through mitotic aberrations that promote expansion of a stem cell-like population. This notion is supported by the following observations: (1) accelerated growth and upregulation of stem cell markers in RUNX1-depleted $\mathrm{ER}^{+}$ breast cancer cells (Fig. 2a-e); (2) increased Sox 2 mRNA levels in Runx1-ablated murine mammary epithelial cells and RUNX1mutant human breast cancer tumour biopsies (Fig. 2f,g); (3) cell cycle-dependent upregulation of P- $\beta$-cat in RUNX1-depleted MCF7 cells (Fig. 6h), which could potentially affect centrosomeanchored microtubule asters and mitotic cell polarity ${ }^{34,56,57}$; (4) loss of cyclin B1 in RUNX1-depleted MCF7 cells (Fig. 6g), potentially reflecting deregulated microtubule organization and premature activation of the anaphase-promoting complex ${ }^{58}$; and (5) abbreviated mitosis/slippage in RUNX1-depleted MCF7 breast cancer cells (Fig. 6a,g). Thus, the increased levels of SOX2, AXIN2 and CD44 (Fig. 2c) do not appear to reflect deregulated LEF/TCF-driven transcription. Instead, they likely represent one aspect of a stem cell-like phenotype, potentially related to changes in centrosomal proteins including $\beta$-catenin and possibly RUNX1 itself $f^{59}$.

The consequences of $\beta$-catenin stimulation in RUNX1depleted $\mathrm{ER}^{+}$luminal cells remain to be fully elucidated. They clearly differ, however, from mechanisms activated by stimulation of the Wnt/ $\beta$-catenin pathway in mouse models where MMTVdriven genetic manipulations lead to breast carcinogenesis ${ }^{38,60-62}$. Such genetic manipulations typically result in the development of basal and alveolar $\mathrm{ER}^{-}$tumours through mechanisms resembling Wnt-driven colon carcinogenesis ${ }^{60-62}$. Possibly, the $\mathrm{ER}^{+}$luminal cell population is spared in these models because it 
a

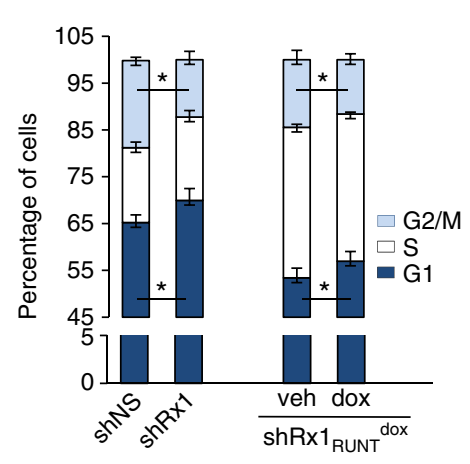

b

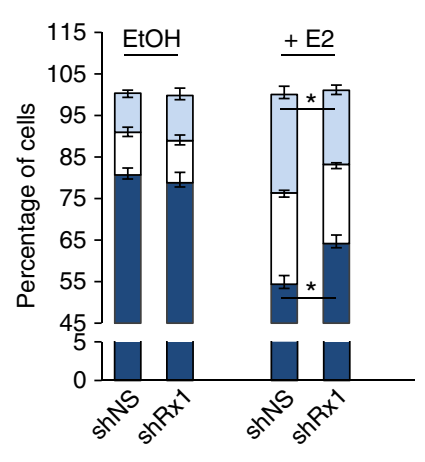

c

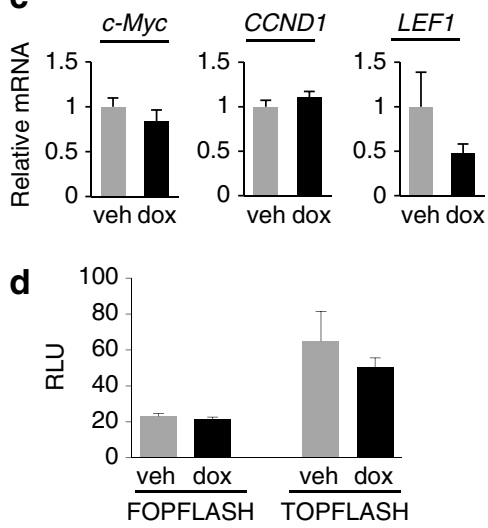

e

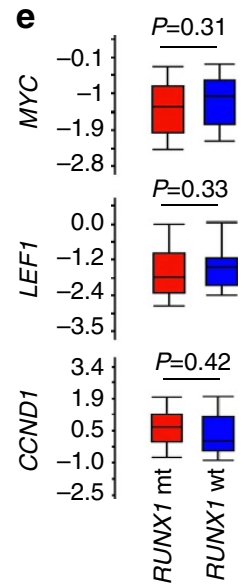

f
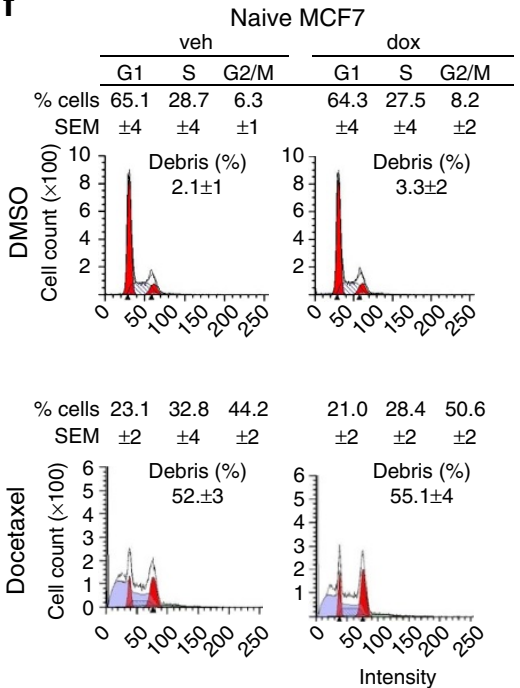

g
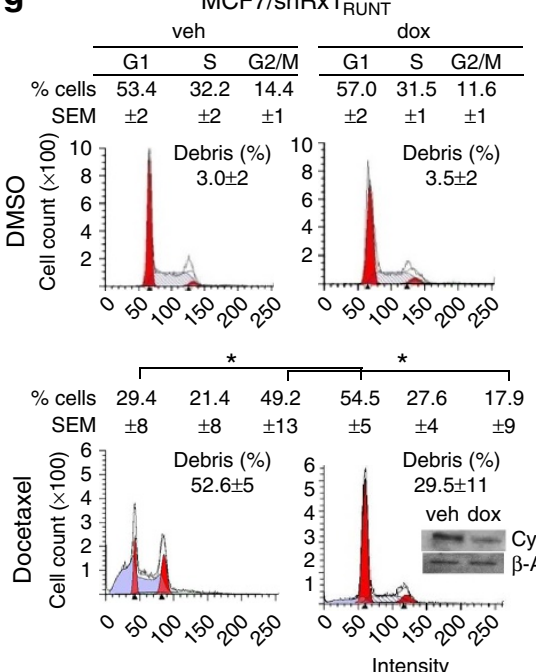

h

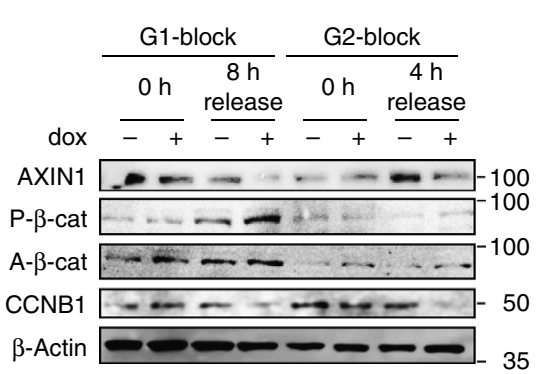

Figure 6 | RUNX1 silencing deregulates breast cancer cell mitosis. RUNX1 was knocked down in MCF7 cells either constitutively (a (left) and b) or conditionally upon treatment with $\operatorname{dox}(\mathbf{a}$ (right), $\mathbf{c}, \mathbf{d} \mathbf{g}$ and $\mathbf{h}$ ). Cells were maintained in medium supplemented with either complete serum (a,c,d,f-h) or CSS (b). (a,b) Cell cycle profiles were obtained by FACS analysis of propidium iodide-stained cells. In $\mathbf{b}$, cells were treated with either vehicle control $(\mathrm{EtOH})$ or estradiol (E2) for $48 \mathrm{~h}$ as indicated. ${ }^{\star} P<0.05$ by $t$-test. (c) RT-qPCR analysis of the indicated Wnt/cell cycle-regulatory genes. Data are corrected for 18S RNA. (d) Luciferase assay of TOPFLASH or control FOPFLASH as a measure of $\beta$-catenin/TCF activity. (e) Expression levels of the indicated genes in RUNX1-mt $(N=17)$ versus RUNX1-wt $(N=389) \mathrm{ER}^{+}$tumours in the breast cancer cohort of TCGA. Significance of the differences was calculated using Mann-Whitney test. (f,g) Naive MCF7 (f) and MCF7/shRx $1_{\text {RUNT }}^{\text {dox }}$ cells ( $\left(\mathbf{g}\right.$ ) were treated with $250 \mathrm{ng} \mathrm{ml}{ }^{-1}$ dox for $72 \mathrm{~h}$ and $2 \mathrm{nM}$ docetaxel was added for the last $48 \mathrm{~h}$ as indicated. Percentages of cells in G1, S and G2/M are given as mean and s.e.m. values from three independent experiments. Representative plots are presented, with inset in $\mathbf{g}$ showing western blot analysis of cyclin B1. (h) MCF7/Rx1sh dox cells were synchronized as described in the 'Methods' section at either G1/S or G2/M, or at the indicated time points. The cells were treated with $250 \mathrm{ng} \mathrm{ml}^{-1}$ dox along with the release from the first thymidine block and extracts were subjected to western blot analysis of the indicated proteins. Quantitative data are mean \pm s.e.m. from three independent experiments. ${ }^{*} P<0.05$ by $t$-test.

lacks $\mathrm{Wnt} / \beta$-catenin-responsive stem cells ${ }^{46}$. Our study suggests that these same $\mathrm{ER}^{+}$cells specifically respond to loss of RUNX1 function by E2-dependent downregulation of AXIN1, and that the mechanisms operative downstream of the RUNX1/AXIN1/ $\beta$-catenin axis in these cells are distinct from those operative in Wnt-driven colon cancer and $\mathrm{ER}^{-}$breast cancer.

Further studies are also warranted to investigate the role of RUNX1 in the control of mammary epithelial cell physiology. We speculate that RUNX1 may prevent undesirable $\beta$-catenin-driven stem cell proliferation in adult $\mathrm{ER}^{+}$luminal cells by antagonizing ER that is activated by circulating oestrogenic compounds and binds at the AXIN1 locus; and, downregulation of RUNX1, as well as RUNX2 and RUNX3, during pregnancy and lactation, and their upregulation during involution ${ }^{13,63}$ may facilitate physiological regulation of $\beta$-catenin in response to hormonal changes during these processes.
Compromised RUNX1 function in $\mathrm{ER}^{+}$breast cancer likely occurs in far more than the $<5 \%$ of tumours with RUNX1 mutations ${ }^{20}$. RUNX1 expression may be compromised in many additional cases, including the $20-40 \%$ invasive ductal carcinomas in which RUNX1 is undetectable by immunohistochemistry (Supplementary Table 1 and refs 12,15). Compromised RUNX1 transcription may be related to promoter hypermethylation (Supplementary Fig. 2), and protein expression and function may also be lost posttranslationally, for example, due to $C B F B$ mutations ${ }^{18-20}$. Subsequent loss of AXIN1 in RUNX1-deficient $\mathrm{ER}^{+}$breast cancer cells may be prevented by treatment with tankyrase inhibitors ${ }^{64,65}$ (Fig. 7), partly alleviating consequences of RUNX1 loss. Tankyrase inhibition would likely be safer than the alternative of restoring RUNX1 itself because RUNX proteins play both tumour suppressor and oncogenic roles in cancer ${ }^{1,2}$. In fact, RUNX1 appears to play an oncogenic rather 
a
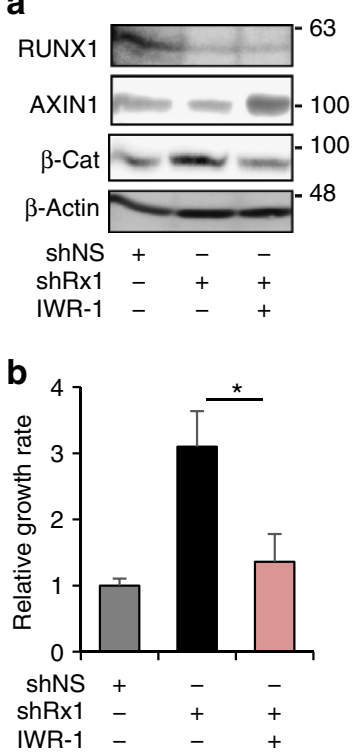

C

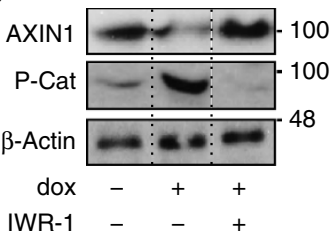

d

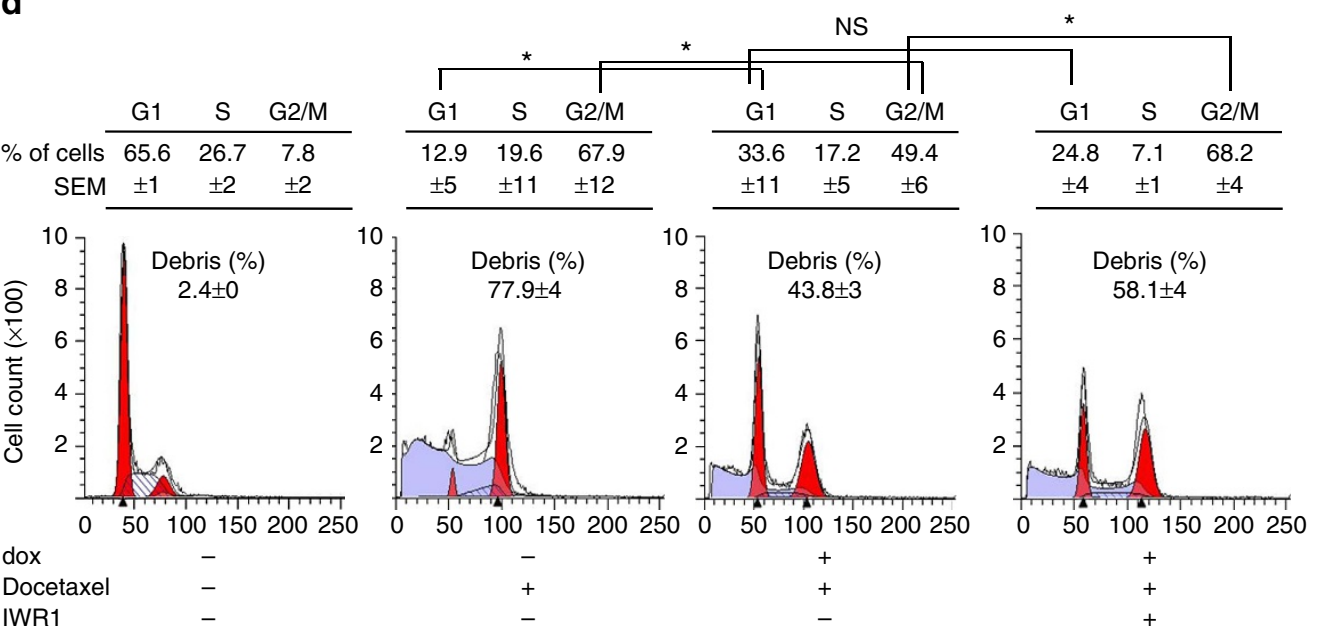

e

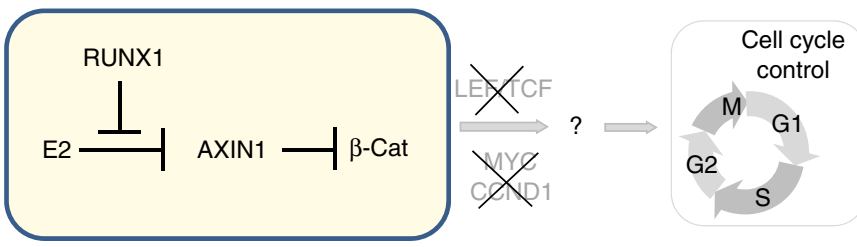

Figure 7 | AXIN1 stabilization normalizes $\boldsymbol{\beta}$-catenin and partially restores cell cycle control in RUNX1-depleted cells. (a) MCF7 cells constitutively expressing shRx1 $1_{3^{\prime}-U T R}(\operatorname{shRx1)}$ were treated for $36 \mathrm{~h}$ with either $5 \mu \mathrm{M}$ IWR1 or its dimethyl sulphoxide vehicle followed by western blot analysis of the indicated proteins. MCF7 expressing a nonspecific shRNA (shNS) were analysed as a reference control. (b) Cells as in a were treated as indicated for 6 days and their growth rate was calculated based on MTT assays as in Fig. $2 \mathrm{~b} .{ }^{\star} P<0.05$ by $t$-test. (c) AXIN1 and P- $\beta$-cat levels were assessed $8 \mathrm{~h}$ after the release of MCF7/shRx d d dox cells from a G1/S double thymidine block as in Fig. 6h. Dox treatment (to silence RUNX1) initiated along with the release from the first thymidine block and IWR1 treatment (to stabilize AXIN1) initiated $17 \mathrm{~h}$ before harvest. (d) MCF7/shRx dox RUNT $_{\text {cells }}$ were treated for $72 \mathrm{~h}$ with dox (to silence RUNX1) and $2 \mathrm{nM}$ docetaxel for $48 \mathrm{~h}$ (to induce mitotic slippage) as in Fig. 6f,g and IWR1 was added for the last $24 \mathrm{~h}$ before FACS analysis. Data are mean \pm s.e.m. $(n=3) .{ }^{\star} P<0.05$ by $t$-test. (e) Working model for the tumour suppressor function of RUNX1 in ER ${ }^{+}$breast cancer, whereby RUNX1 prevents E2-mediated AXIN1 suppression. Mechanisms linking the RUNX1/AXIN1/ $\beta$-catenin axis to loss of cell cycle control in RUNX1-deficient ER ${ }^{+}$mammary epithelial cells remain to be fully elucidated. They entail stimulation of neither LEF/TCF, nor c-MYC, nor CCND1, nor G1/S phase transition, but are associated instead with deregulated mitosis.

than a tumour suppressor role in triple-negative breast cancer $^{12,15}$, possibly, in part, related to alternative splicing of AXIN1 (ref. 66). In $\mathrm{ER}^{+}$breast cancer, however, RUNX1 predominantly functions as a tumour suppressor, and the present work attributes this function at least in part to antagonism of oestrogen-mediated AXIN1 suppression.

\section{Methods}

Clinical data mining. High-throughput whole-genome data used in this study was from cases for which clinical information was available in the breast cancer cohort of TCGA ${ }^{20}$. Gene expression (based on either microarray hybridization or RNA-seq), DNA methylation and somatic mutation data were retrieved from the TCGA Data Portal (http://cancergenome.nih.gov/). In addition, RNA-seq data were downloaded from the UCSC Cancer Genomics Browser (http://genomecancer.ucsc.edu) as ranked expression scores. Expression microarray data and RUNX1 mutation status of $209 \mathrm{ER}^{+}$tumours described by Ellis et al. ${ }^{18}$ was obtained from the University of North Carolina Microarray Database.

Mice. Mice used in this study have been previously described ${ }^{13}$. YFP ${ }^{+} \mathrm{ER}^{-}$ luminal progenitor cells (LPs) were sorted from MMTV-Cre;Runx $1^{f / f} ; R 26 Y$ and $M M T V-C r e ; R u n x 1^{+/+} ; R 26 Y$ (control) females (2 months of age) and $\mathrm{YFP}^{+} \mathrm{ER}^{+}$ mature luminal cells (MLs) were sorted from MMTV-Cre;Runx $1^{\mathrm{fff} ;} ; \mathrm{Rb} 1^{\mathrm{fff}} ; R 26 Y$ and $M M T V-C r e ; R u n x 1^{+/+} ; R b 1^{f f f} ; R 26 Y$ (control) females (either 2-month or 7-month old). $R 26 Y$ is a conditional Cre-reporter that expresses YFP upon Cre-mediated recombination. In the compound mice, conditional knockout of Runx1 (and Rb1) in mammary epithelial cells is linked to YFP expression. Breeding with $R b 1^{\mathrm{f} / f}$ mice facilitated rescue of the $\mathrm{ER}^{+}$luminal cell subpopulation upon $R b 1$ deletion as described ${ }^{13}$. FACS sorting was performed with a FACSAria sorter (BD Biosciences) using antibodies from eBiosciences including CD24-eFluor450, CD24-eFluor605, CD29-APC, c-Kit-PE-CY7, CD14-PE and biotinylated CD31, CD45, TER119 and Streptavidin-PerCP-CY5.5. All animal work was performed in strict accordance with the recommendations in the Guide for the Care and Use of Laboratory Animals of the National Institutes of Health and was approved by the Institutional Animal Care and Use Committee of Boston Children's Hospital where the animals are housed.

Cells. The ER ${ }^{+}$MCF7 and T47D, as well as the ER ${ }^{-}$MDA-MB-231 breast cancer cells were from the American Type Culture Collection. MCF10A cells were obtained from the Karmanos Cancer Institute (Detroit, Michigan). MCF7 and T47D cells were cultured in Dulbecco's Modified Eagle's medium (DMEM; Mediatech, Inc) and RPMI-1640 medium (Mediatech, Inc), respectively, both supplemented with $10 \%$ fetal bovine serum (FBS; Gemini Bio-products).

MDA-MB-231 cells were cultured in DMEM/F12 (Mediatech, Inc) supplemented with 5\% FBS. MCF10A cells were cultured in DMEM/F12 supplemented with 5\% horse serum (Gemini Bio-products), $10 \mu \mathrm{g} \mathrm{ml}^{-1}$ insulin (Sigma-Aldrich),

$20 \mathrm{ng} \mathrm{ml}^{-1}$ EGF (Sigma-Aldrich), $0.5 \mu \mathrm{g} \mathrm{ml}^{-1}$ hydrocortisone (Sigma-Aldrich) and $0.1 \mathrm{M} \mathrm{CaCl}_{2}$. For oestrogen treatment, the cells were washed three times with phosphate-buffered saline and maintained for $48 \mathrm{~h}$ in phenol-red-free growth medium supplemented with 10\% CSS (Gemini Bio-products), followed by estradiol (E2) administration (Sigma-Aldrich). ICI 182780, IWR1 and docetaxel, also from Sigma-Aldrich, were added to the culture medium as indicated. 
In vitro RUNX1 manipulation. Mission shRNA lentiviral plasmids targeting either the RUNT or the 3'-UTR of RUNX1 were purchased from Sigma-Aldrich (Supplementary Table 2). For packaging, the plasmids were co-transfected into HEK293T cells along with helper plasmids pMD.G1 and pCMV.R8.91 using the calcium chloride method. Culture media containing viral particles were harvested after $48-72 \mathrm{~h}$ and used for the transduction of breast cancer cells in the presence of $8 \mu \mathrm{g} \mathrm{ml}^{-1}$ polybrene (Millipore Corp., MA, USA) followed by selection with $1 \mu \mathrm{g} \mathrm{ml}^{-1}$ puromycin for 5 days. Alternatively, we used the pSLIK vector system ${ }^{67}$ for conditional RUNX1 silencing. Lentiviruses containing dox-inducible shRNAs that target either the RUNT domain ( $\operatorname{shRx} 1_{\text {RUNT }}^{\text {dox }}$ ) or the $3^{\prime}$-UTR $\left(\operatorname{shRx} 1_{3^{\prime}-\text { UTR }}^{\text {dox }}\right)$ of RUNX1 (Supplementary Table 2) were engineered by first cloning the shRNAs into the entry vector pEN_TmiRc3. The resulting plasmids were each recombined using Gateway LR Clonase II enzyme mix (Invitrogen) with the pSLIK destination vector carrying a neomycin-resistant gene. Transduced cells were selected with Geneticin (Gemini Bio-products) at $1 \mathrm{mg} \mathrm{ml}^{-1}$ for MCF7 cells and $0.4 \mathrm{mg} \mathrm{ml}^{-1}$ for T47D cells. The pSLIK vector system was also used to conditionally express FLAG-tagged RUNX1, either wild type or mutant, by following the protocol previously described for conditional expression of FLAG-tagged RUNX2 (refs 9,68). For RUNX1 rescue experiments, cells containing dox-inducible FLAG-RUNX1 $\left(\mathrm{Rx}^{\mathrm{dox}}\right)$ were additionally transduced with a lentivirus expressing the Mission shRNA against the 3'-UTR of RUNX1.

\section{Cell proliferation and cell cycle assays. Cell proliferation was assessed} using 3-(4,5-dimethylthiazol-2-yl)-2,5-diphenyltetrazolium bromide (MTT) colorimetric assay. Approximately 5,000 cells per well were seeded in 96-well tissue culture plates and incubated on the indicated days with $0.5 \mathrm{mg} \mathrm{ml}^{-1}$ MTT (Sigma-Aldrich) for $3 \mathrm{~h}$ before lysis with dimethyl sulphoxide. Absorbance at a 595 $\mathrm{nm}$ wavelength was measured using Victor $_{3} \mathrm{~V}$ plate reader from PerkinElmer. Cell cycle synchronization at G1/S was achieved by double-thymidine block. The cells were incubated for $16 \mathrm{~h}$ in medium containing $2 \mathrm{mM}$ thymidine (Sigma-Aldrich), released for 8-12 h in DMEM containing 10\% FBS, and then incubated again in $2 \mathrm{mM}$ thymidine for $16-18 \mathrm{~h}$. For G2/M synchronization, the cells were released from the double-thymidine block for $4 \mathrm{~h}$ before treatment with $100 \mathrm{nM}$ nocodazole (Sigma-Aldrich) for $14 \mathrm{~h}$. Percentage of cells in the various phases of the cell cycle was quantified by propidium iodide staining and flow cytometry using LSRII flow cytometer (BD Biosciences) and the Modfit LT SynchWizard Tool (Verity Software House).

TOPFLASH assay. The cells were seeded at a density of 50,000 per well in 24-well plates, and LEF/TCF-mediated transcriptional activity was measured using the Super8xTOPFLASH reporter plasmid with the Super8xFOPFLASH plasmid serving as control. Plasmids were transiently transfected using the jetPrime transfection reagent (Polyplus-Transfection) according to the manufacturer's specification. Luciferase activity was determined with the Dual-Luciferase Reporter Assay System (Promega) using a Victor ${ }_{3} \mathrm{~V}$ plate reader (PerkinElmer).

RT-qPCR. Total RNA from cultured cells was isolated using Aurum Total RNA mini-kit (Bio-Rad) and cDNA was synthesized from $1 \mu \mathrm{g}$ of total RNA with iScript reverse transcription kit (Bio-Rad). Quantitative real-time PCR was carried out in triplicate using a CFX96 instrument (Bio-Rad) and the Maxima SYBR Green/ Fluorescein Master Mix (ThermoFisher Scientific). Relative mRNA expression values were normalized to those of GAPDH and/or 18S RNA. For cell populations isolated from mouse tissue, the RNA was extracted using RNeasy kit (Qiagen) and amplified with the Ovation RNA Amplification System V2 kit (Nugen). cDNA was synthesized with Omniscript (Qiagen) according to the manufacturer's protocol, and real-time qPCR was performed using FastStart SYBR Green Master (Roche). Gene expression in $\mathrm{ER}^{-} \mathrm{LPs}$ was measured in LPs sorted from MMTV-Cre;Runx $\mathrm{f}^{\mathrm{f}}$; $R 26 Y$ and MMTV-Cre;Runx $1^{+/+} ; R 26 Y$ (control) adult females. Since the $\mathrm{ER}^{+} \mathrm{ML}$ subpopulation was largely lost in $M M T V-C r e ; R u n x 1^{f / f} ; R 26 Y$ females $^{13}$, gene expression in $\mathrm{ER}^{+}$MLs was measured in rescued MLs (by simultaneous inactivation of $R b 1$ together with Runx1 (ref. 13) sorted from MMTV-Cre;Runx $1^{f / f} ; R b 1^{f / f} ; R 26 Y$ female mice (7 months of age), upon normalization to those in $\mathrm{ER}^{+}$MLs sorted from 2-month-old MMTV-Cre;Runx ${ }^{f / f} ; R b 1^{f / f} ; R 26 Y$ female mice (to correct for changes in cell compositions in the ML FACS gate) and to those in the corresponding ML subpopulations sorted from 7-month- and 2-month-old MMTV-Cre;Runx1 ${ }^{+/+}$; $R b 1^{f / f} ; R 26 Y$ control females, respectively. Primers used for RT-qPCR amplifications are listed in Supplementary Table 2.

Western blot analysis. The cells were lysed using RIPA buffer (ThermoFisher Scientific) supplemented with protease (Sigma-Aldrich) and phosphatase inhibitors (ThermoFisher Scientific). Cytoplasmic and nuclear extracts were prepared using NE-PER kit (ThermoFisher Scientific) following the manufacturer's instructions. Proteins were resolved by SDS-PAGE (polyacrylamide gel electrophoresis) and transferred to AmershamHybond-P PVDF membranes (GE Healthcare), followed by blocking with $5 \%$ non-fat dry milk. The following antibodies were purchased from Cell Signaling Technology, Inc. and used at a 1:1,000 dilution: rabbit antiRUNX1 (\#8529), rabbit-anti-AXIN1 (\#2087), rabbit anti-phospho- $\beta$-catenin (Ser33/37/Thr41; \#9561) and mouse anti-CCNB1 (\#4135). Mouse anti-active$\beta$-catenin (\#05-665) was purchased from Millipore and used at a 1:1,000 dilution.
Rabbit anti- $\beta$-catenin (ab32572) and rabbit anti-SOX2 from Abcam were used at a 1:5,000 dilution. Mouse anti-FLAG (F3165) antibody from Sigma-Aldrich was used at a 1:2,000 dilution. Goat anti-Actin (sc-1616) and goat anti-GAPDH (sc-20357) antibodies were purchased from Santa Cruz Biotechnology and used at a 1:200 dilutions. The mouse monoclonal anti- $\beta$-tubulin antibody, developed by Dr Charles Walsh, was obtained from the Developmental Studies Hybridoma Bank under the auspices of the NICHD and The University of Iowa, Department of Biological Sciences, Iowa City, USA, and used at a 1:5,000 dilution. HRPconjugated donkey anti-goat (sc-2033), donkey anti-mouse (sc-2314) and goat anti-rabbit (sc-2004) antibodies were purchased from Santa Cruz Biotechnology and used at a 1:2,000 dilution. Immunodetection was performed using Pierce ECL2 western blotting detection system (ThermoFisher Scientific). Uncropped scans of key western blots are provided as a Supplementary Fig. 10.

Immunohistochemistry. TMA slides (TM-1007) were purchased from Protein Biotechnologies, Inc. The majority of tumours represented on this TMA were invasive ductal carcinomas, of which 31 were $\mathrm{ER}^{+}$and 33 were $\mathrm{ER}^{-}$. TMAs were deparaffinized and rehydrated before antigen retrieval in $10 \mathrm{mM}$ sodium citrate buffer at $95^{\circ} \mathrm{C}$. After cool-down, the slides were incubated for 20 min in $1 \% \mathrm{H}_{2} \mathrm{O}_{2}$ in methanol to block endogenous peroxidase, washed with TBS and blocked with Background Punisher (\#BP974, Biocare Medical) for $10 \mathrm{~min}$ at room temperature. The slides were then incubated overnight at $4{ }^{\circ} \mathrm{C}$ with antibodies against RUNX1 (\#8529) or AXIN1 (\#2087), both purchased from Cell Signaling Technology and used at a 1:50 dilution. Afterwards, the slides were washed with TBS, and treated with MACH4 Universal HRP-polymer (\#M4U534, Biocare Medical) for $30 \mathrm{~min}$. After 5-min incubation with a DAB reagent (\#K3466, DAKO) to visualize HRP, the slides were rinsed, counterstained with haematoxylin and topped with cover slips. ER histoscores were provided by the manufacturer and presence or absence of RUNX1 and AXIN1 was determined by a certified surgical pathologist (P.M.-F.) The association between the RUNX1 status and AXIN1 status was tested using the Pearson chi-square test for the $2 \times 2$ table, for ER ${ }^{\text {low }}$ and $\mathrm{ER}^{\text {high }}$ tumours separately. Odds ratios for the $\mathrm{ER}^{\text {low }}$ and $\mathrm{ER}^{\text {high }}$ tumours were compared using the Breslow-Day test for homogeneity of odds ratios.

Expression profiling and ChIP-seq. MCF7/shRx1 and MCF7/shNS cells, expressing shRNAs that target RUNX1 or a nonspecific shRNA, respectively, were maintained in CSS for $48 \mathrm{~h}$ before treatment with either $10 \mathrm{nM}$ E2 or the ethanol vehicle for $48 \mathrm{~h}$. Total RNA was extracted using Aurum Total RNA mini-kit (Bio-Rad) and global expression profiling was performed using BeadChips (Illumina) by the Southern California Genotyping Consortium at UCLA. To compare the RUNX1 and the RUNX2 transcriptomes, MCF7 cells were first transduced with the respective dox-inducible pSLIK vectors, and then with the Mission $\operatorname{shRx} 1_{3^{\prime} \text {-UTR }}$ vector. RUNX1 depleted cells were then treated with $0.25 \mu \mathrm{g} \mathrm{ml}^{-1}$ dox to induce RUNX1 or RUNX2 expression and/or with $10 \mathrm{nM} \mathrm{E2}$ for $48 \mathrm{~h}$, and global mRNA expression was profiled as described above. RUNX1 ChIP-seq was performed essentially as described ${ }^{69}$. Briefly, MCF7 cells were crosslinked, lysed and sonicated to obtain DNA fragments mostly in the 200-1,000-bp range. Immunoprecipitation was performed at $4{ }^{\circ} \mathrm{C}$ overnight with anti-RUNX1 antibody (ab23980, Abcam). Downstream analysis was performed on high-fidelity peaks reproduced in two independent experiments. GEO accession numbers for the microarray and ChIP-Seq data are GSE65620, GSE65616 and GSE65313. MCF7 $\mathrm{ER} \alpha$ ChIP-seq data was from GSE14664. ChIP-qPCR validation was performed using antibodies against either RUNX1 (ab23980, Abcam) or ER $\alpha$ (sc543x from Santa Cruz Biotechnology). For ER $\alpha$, ChIP-qPCR cells were maintained in CSS for $48 \mathrm{~h}$ and treated with $10 \mathrm{nM}$ E2 for $1 \mathrm{~h}$. A region near GAPDH served as negative control.

Data analysis. Global gene expression raw data from MCF7 cell cultures was processed using GenomeStudio (Illumina Inc). After background subtraction and quantile normalization, the signal intensity values were exported to the Partek Genomics Suite 6.4 (Partek, Inc.) using 'Partek's Report Plug-in'option in the GenomeStudio software and differential expression was analysed by one-way analysis of variance. Differentially expressed genes were investigated using the Ingenuity Pathways Analysis package ( http://www.ingenuity.com). Fisher's exact test as implemented in the Ingenuity Pathways Analysis software was used to calculate $P$ values. To estimate RUNX1 inhibitory activity on the basis of RNA-seq tumour data mining, we first defined a set of 123 genes that were expressed in E2treated MCF7/shRx1 $1_{3^{\prime} \text {-UTR }}$ cells at levels $\geq 1.7$-fold higher $(P<0.05)$ than the respective expression levels in MCF7/shNS cells (Fig. 3a). The ranked expression score for each of these gene in each tumour in the breast cancer cohort of TCGA was obtained from the UCSC Cancer Genomics Browser. We then defined an 'inhibitory index' for RUNX1 in each tumour as the mean rank for the 123 genes and calculated its correlation with the respective AXIN1 expression level. For the analysis of RUNX1 locations, the ChIP-seq reads from biological replicates and an input control library were aligned to the hg19 build of the human genome. Peaks for RUNX1, as well as for ER $\alpha$ (based on Welboren et al. ${ }^{47}$ ) were called with $\mathrm{MACS}^{70}$ with default parameters using input as control and only reproducible peaks in both RUNX1 replicates were retained for further analysis. Unless 
otherwise stated, statistical significance of differences in this study was determined using InStat 3.0b GraphPad Software (GraphPad Software, Inc., CA, USA).

\section{References}

1. Blyth, K., Cameron, E. R. \& Neil, J. C. The RUNX genes: gain or loss of function in cancer. Nat. Rev. Cancer 5, 376-387 (2005).

2. Chimge, N. O. \& Frenkel, B. The RUNX family in breast cancer: relationships with estrogen signaling. Oncogene 32, 2121-2130 (2013).

3. Ito, Y., Bae, S. C. \& Chuang, L. S. The RUNX family: developmental regulators in cancer. Nat. Rev. Cancer 15, 81-95 (2015).

4. Chen, L. F. Tumor suppressor function of RUNX3 in breast cancer. J. Cell. Biochem. 113, 1470-1477 (2012).

5. Chuang, L. S. \& Ito, Y. RUNX3 is multifunctional in carcinogenesis of multiple solid tumors. Oncogene 29, 2605-2615 (2010).

6. Huang, B. et al. RUNX3 acts as a tumor suppressor in breast cancer by targeting estrogen receptor alpha. Oncogene 31, 527-534 (2012).

7. Khalid, O. et al. Modulation of Runx2 activity by estrogen receptor-alpha: implications for osteoporosis and breast cancer. Endocrinology 149, 5984-5995 (2008).

8. Chimge, N. O. et al. Regulation of breast cancer metastasis by Runx2 and estrogen signaling: the role of SNAI2. Breast. Cancer Res. 13, R127 (2011)

9. Chimge, N. O. et al. Opposing effects of Runx2 and estradiol on breast cancer cell proliferation: in vitro identification of reciprocally regulated gene signature related to clinical letrozole responsiveness. Clin. Cancer Res. 18, 901-911 (2012).

10. Pratap, J., Lian, J. B. \& Stein, G. S. Metastatic bone disease: role of transcription factors and future targets. Bone 48, 30-36 (2011).

11. Janes, K. A. RUNX1 and its understudied role in breast cancer. Cell Cycle 10, 3461-3465 (2011).

12. Browne, G. et al. Runx1 is associated with breast cancer progression in MMTVPyMT transgenic mice and its depletion in vitro inhibits migration and invasion. J. Cell. Physiol. 30, 2522-2532 (2015).

13. van Bragt, M. P., Hu, X., Xie, Y. \& Li, Z. RUNX1, a transcription factor mutated in breast cancer, controls the fate of ER-positive mammary luminal cells. Elife 4, e03881 (2014).

14. Taniuchi, I., Osato, M. \& Ito, Y. Runx1: no longer just for leukemia. EMBO J. 31, 4098-4099 (2012).

15. Ferrari, N. et al. Expression of RUNX1 correlates with poor patient prognosis in triple negative breast cancer. PLoS ONE 9, el00759 (2014).

16. Kadota, M. et al. Delineating genetic alterations for tumor progression in the MCF10A series of breast cancer cell lines. PLoS ONE 5, e9201 (2010).

17. Wang, L., Brugge, J. S. \& Janes, K. A. Intersection of FOXO- and RUNX1mediated gene expression programs in single breast epithelial cells during morphogenesis and tumor progression. Proc. Natl Acad. Sci. USA 108, E803-E812 (2011)

18. Ellis, M. J. et al. Whole-genome analysis informs breast cancer response to aromatase inhibition. Nature 486, 353-360 (2012).

19. Banerji, S. et al. Sequence analysis of mutations and translocations across breast cancer subtypes. Nature 486, 405-409 (2012).

20. TCGA. Comprehensive molecular portraits of human breast tumours. Nature 490, 61-70 (2012)

21. Dawson, S. J., Rueda, O. M., Aparicio, S. \& Caldas, C. A new genome-driven integrated classification of breast cancer and its implications. EMBO J. 32, 617-628 (2013)

22. Germain, D. Estrogen carcinogenesis in breast cancer. Endocrinol. Metab. Clin. North Am. 40, 473-484 vii (2011).

23. Clemons, M. \& Goss, P. Estrogen and the risk of breast cancer. N. Engl. J. Med. 344, 276-285 (2001).

24. Pike, M. C., Spicer, D. V., Dahmoush, L. \& Press, M. F. Estrogens, progestogens, normal breast cell proliferation, and breast cancer risk. Epidemiol. Rev. 15 17-35 (1993).

25. Hurtado, A., Holmes, K. A., Ross-Innes, C. S., Schmidt, D. \& Carroll, J. S. FOXA1 is a key determinant of estrogen receptor function and endocrine response. Nat. Genet. 43, 27-33 (2011).

26. Fiorito, E., Katika, M. R. \& Hurtado, A. Cooperating transcription factors mediate the function of estrogen receptor. Chromosoma 122, 1-12 (2013)

27. Asselin-Labat, M. L. et al. Gata-3 is an essential regulator of mammary-gland morphogenesis and luminal-cell differentiation. Nat. Cell Biol. 9, 201-209 (2007).

28. Tan, S. K. et al. AP-2gamma regulates oestrogen receptor-mediated long-range chromatin interaction and gene transcription. EMBO J. 30, 2569-2581 (2011).

29. Stender, J. D. et al. Genome-wide analysis of estrogen receptor alpha DNA binding and tethering mechanisms identifies Runxl as a novel tethering factor in receptor-mediated transcriptional activation. Mol. Cell. Biol. 30, 3943-3955 (2010).

30. Stamos, J. L. \& Weis, W. I. The beta-catenin destruction complex. Cold Spring Harb. Perspect. Biol. 5, a007898 (2013).
31. Clevers, H. Wnt/beta-catenin signaling in development and disease. Cell 127, 469-480 (2006).

32. Li, V. S. et al. Wnt signaling through inhibition of beta-catenin degradation in an intact Axin1 complex. Cell 149, 1245-1256 (2012).

33. Huang, P., Senga, T. \& Hamaguchi, M. A novel role of phospho-beta-catenin in microtubule regrowth at centrosome. Oncogene 26, 4357-4371 (2007).

34. Mbom, B. C., Nelson, W. J. \& Barth, A. beta-catenin at the centrosome: discrete pools of beta-catenin communicate during mitosis and may co-ordinate centrosome functions and cell cycle progression. Bioessays 35, 804-809 (2013).

35. Kaplan, D. D., Meigs, T. E., Kelly, P. \& Casey, P. J. Identification of a role for beta-catenin in the establishment of a bipolar mitotic spindle. J. Biol. Chem. 279, 10829-10832 (2004)

36. Mbom, B. C., Siemers, K. A., Ostrowski, M. A., Nelson, W. J. \& Barth, A. I. Nek2 phosphorylates and stabilizes beta-catenin at mitotic centrosomes downstream of Plk1. Mol. Biol. Cell 25, 977-991 (2014).

37. Alexander, C. M., Goel, S., Fakhraldeen, S. A. \& Kim, S. Wnt signaling in mammary glands: plastic cell fates and combinatorial signaling. Cold Spring Harb. Perspect. Biol. 4, a008037 (2012).

38. Incassati, A., Chandramouli, A., Eelkema, R. \& Cowin, P. Key signaling nodes in mammary gland development and cancer: beta-catenin. Breast Cancer Res. 12, 213 (2010)

39. Reya, T. \& Clevers, H. Wnt signalling in stem cells and cancer. Nature $\mathbf{4 3 4}$, 843-850 (2005)

40. Ring, A., Kim, Y. M. \& Kahn, M. Wnt/catenin signaling in adult stem cell physiology and disease. Stem Cell Rev. 10, 512-525 (2014).

41. Clevers, H., Loh, K. M. \& Nusse, R. Stem cell signaling. An integral program for tissue renewal and regeneration: Wnt signaling and stem cell control. Science 346, 1248012 (2014).

42. Marcato, P. et al. Aldehyde dehydrogenase activity of breast cancer stem cells is primarily due to isoform ALDH1A3 and its expression is predictive of metastasis. Stem Cells 29, 32-45 (2011).

43. Lu, X., Mazur, S. J., Lin, T., Appella, E. \& Xu, Y. The pluripotency factor nanog promotes breast cancer tumorigenesis and metastasis. Oncogene 33, 2655-2664 (2014).

44. Guo, W. Concise review: breast cancer stem cells: regulatory networks, stem cell niches, and disease relevance. Stem Cells Transl. Med. 3, 942-948 (2014).

45. Leis, O. et al. Sox 2 expression in breast tumours and activation in breast cancer stem cells. Oncogene 31, 1354-1365 (2012).

46. van Amerongen, R., Bowman, A. N. \& Nusse, R. Developmental stage and time dictate the fate of $\mathrm{Wnt} /$ beta-catenin-responsive stem cells in the mammary gland. Cell Stem Cell 11, 387-400 (2012).

47. Welboren, W. J. et al. ChIP-Seq of ERalpha and RNA polymerase II defines genes differentially responding to ligands. EMBO J. 28, 1418-1428 (2009).

48. Martin, A. et al. Estrogens antagonize RUNX2-mediated osteoblast-driven osteoclastogenesis through regulating RANKL membrane association. Bone 75, 96-104 (2015).

49. Clevers, H. \& Nusse, R. Wnt/beta-catenin signaling and disease. Cell 149 1192-1205 (2012).

50. Holland, J. D., Klaus, A., Garratt, A. N. \& Birchmeier, W. Wnt signaling in stem and cancer stem cells. Curr. Opin. Cell Biol. 25, 254-264 (2013).

51. Hernandez-Vargas, H., Palacios, J. \& Moreno-Bueno, G. Molecular profiling of docetaxel cytotoxicity in breast cancer cells: uncoupling of aberrant mitosis and apoptosis. Oncogene 26, 2902-2913 (2007)

52. Morse, D. L., Gray, H., Payne, C. M. \& Gillies, R. J. Docetaxel induces cell death through mitotic catastrophe in human breast cancer cells. Mol. Cancer Ther. 4, 1495-1504 (2005).

53. Olmeda, D., Castel, S., Vilaro, S. \& Cano, A. Beta-catenin regulation during the cell cycle: implications in G2/M and apoptosis. Mol. Biol. Cell 14, 2844-2860 (2003).

54. Lee, E., Salic, A., Kruger, R., Heinrich, R. \& Kirschner, M. W. The roles of APC and Axin derived from experimental and theoretical analysis of the Wnt pathway. PLoS Biol. 1, E10 (2003).

55. Chen, B. et al. Small molecule-mediated disruption of Wnt-dependent signaling in tissue regeneration and cancer. Nat. Chem. Biol. 5, 100-107 (2009).

56. Ligon, L. A., Karki, S., Tokito, M. \& Holzbaur, E. L. Dynein binds to betacatenin and may tether microtubules at adherens junctions. Nat. Cell Biol. 3, 913-917 (2001).

57. Chilov, D. et al. Phosphorylated beta-catenin localizes to centrosomes of neuronal progenitors and is required for cell polarity and neurogenesis in developing midbrain. Dev. Biol. 357, 259-268 (2011).

58. Brito, D. A. \& Rieder, C. L. Mitotic checkpoint slippage in humans occurs via cyclin B destruction in the presence of an active checkpoint. Curr. Biol. 16, 1194-1200 (2006).

59. Chuang, L. S. et al. RUNX3 interactome reveals novel centrosomal targeting of RUNX family of transcription factors. Cell Cycle 11, 1938-1947 (2012).

60. Imbert, A., Eelkema, R., Jordan, S., Feiner, H. \& Cowin, P. Delta N89 betacatenin induces precocious development, differentiation, and neoplasia in mammary gland. J. Cell Biol. 153, 555-568 (2001). 
61. Teissedre, B. et al. MMTV-Wnt1 and -DeltaN89beta-catenin induce canonical signaling in distinct progenitors and differentially activate Hedgehog signaling within mammary tumors. PLoS ONE 4, e4537 (2009).

62. Tsukamoto, A. S., Grosschedl, R., Guzman, R. C., Parslow, T. \& Varmus, H. E. Expression of the int-1 gene in transgenic mice is associated with mammary gland hyperplasia and adenocarcinomas in male and female mice. Cell 55, 619-625 (1988).

63. Blyth, K. et al. Runx2 in normal tissues and cancer cells: a developing story. Blood Cells Mol. Dis. 45, 117-123 (2010).

64. Lehtio, L., Chi, N. W. \& Krauss, S. Tankyrases as drug targets. FEBS J. 280, 3576-3593 (2013).

65. Huang, S. M. et al. Tankyrase inhibition stabilizes axin and antagonizes Wnt signalling. Nature 461, 614-620 (2009).

66. Zhang, X. et al. Mechanistic insight into Myc stabilization in breast cancer involving aberrant Axin1 expression. Proc. Natl Acad. Sci. USA 109, 2790-2795 (2012).

67. Shin, K. J. et al. A single lentiviral vector platform for microRNA-based conditional RNA interference and coordinated transgene expression. Proc. Natl Acad. Sci. USA 103, 13759-13764 (2006).

68. Baniwal, S. K. et al. Runx2 transcriptome of prostate cancer cells: insights into invasiveness and bone metastasis. Mol. Cancer 9, 258 (2010).

69. Little, G. H. et al. Genome-wide Runx2 occupancy in prostate cancer cells suggests a role in regulating secretion. Nucleic Acids Res. 40, 3538-3547 (2012).

70. Zhang, Y. et al. Model-based analysis of ChIP-Seq (MACS). Genome Biol. 9, R137 (2008).

\section{Acknowledgements}

We acknowledge NIH grants RO1 DK07112 and RO1 DK07112S from the National Institute of Diabetes and Digestive and Kidney Diseases (NIDDK) to B.F., who holds the J. Harold and Edna L. LaBriola Chair in Genetic Orthopedic Research at USC. This work was also supported by an award from the SC CTSI Pilot Funding Program to N.C. and D.T. Support to Z.L. was from NIH grants R00 CA126980 and R01 HL107663, and from Harvard Stem Cell Institute Seed Grant SG-0062-10-01. Support to C.L. was from the Margaret Early Trustee Foundation, the American Cancer Society (RSG-11-121-01CCG), and the National Institutes of Health (R01 CA140964). Support for S.G. was by grant P30CA014089 from the National Cancer Institute. We thank Michael Kahn, Louis Dubeau, Michael Stallcup and Meng Li of USC, as well as our patient advocates Mary Aalto and Ghecemy Lopez, for insightful discussions, and Zea Borok for the TOPFLASH and FOPFLASH plasmids. The microarray analysis was performed at the Southern
California Genotyping Consortium at UCLA http://scgc.genetics.ucla.edu/ under the direction of Joseph DeYoung. We are also thankful to Charles Nicolet at the USC/Norris Epigenome Center for high throughput sequencing; Lora Barski at the USC Broad Center Flow Cytometry Core and Lian Asatryan and Maira Soto at the USC Translational Research Laboratory for their help with FACS analysis. Work at the USC Flow Cytometry Core Facility, the USC Epigenome Center and the USC Biostatistics Core was support by grant P30CA014089 from the National Cancer Institute and the USC Office of the Provost, Dean's Development Funds, Keck School of Medicine of USC.

\section{Author contributions}

N.C., D.T., M.J.E. and B.F. conceived the study. N.C., G.H.L., S.K.B, H.A., A.O.L., Z.Y.L Y.X., T.Z., A.M. and P.U. performed the experiments. P.M.-F. performed the tissue microarray scoring. N.C., S.G., D.E.S. and B.F. analysed the data. N.C., C.L., Z.L., M.J.E., D.T., D.E.S. and B.F. wrote the manuscript.

\section{Additional information}

Accession codes: Microarray and ChIP-Seq data have been deposited in the GEO under accession codes GSE65620, GSE65616 and GSE65313.

Supplementary Information accompanies this paper at http://www.nature.com/ naturecommunications

Competing financial interests: The authors declare no competing financial interests.

Reprints and permission information is available online at http://npg.nature.com/ reprintsandpermissions/

How to cite this article: Chimge, N. et al. RUNX1 prevents oestrogen-mediated AXIN1 suppression and $\beta$-catenin activation in ER-positive breast cancer. Nat. Commun. 7:10751 doi: 10.1038/ncomms10751 (2016).

(c) (i) This work is licensed under a Creative Commons Attribution 4.0 International License. The images or other third party material in this article are included in the article's Creative Commons license, unless indicated otherwise in the credit line; if the material is not included under the Creative Commons license, users will need to obtain permission from the license holder to reproduce the material. To view a copy of this license, visit http://creativecommons.org/licenses/by/4.0/ 